\title{
Convergence of the split-step $\theta$-method for stochastic age-dependent population equations with Markovian switching and variable delay
}

\author{
Shounian Denga, ${ }^{\mathrm{a} b}$, Weiyin Fei ${ }^{\mathrm{b}, *}$, Yong Liang ${ }^{\mathrm{c}}$, Xuerong Mao ${ }^{\mathrm{d}}$ \\ ${ }^{a}$ School of Science, Nanjing University of Science and Technology, Nanjing, Jiangsu 210094, China \\ ${ }^{b}$ School of Mathematics and Physics, Anhui Polytechnic University, Wuhu, Anhui 24100, China \\ ${ }^{c}$ School of Mathematics, Shanghai University of Finance and Economics, Shanghai, 200433, China \\ ${ }^{d}$ Department of Mathematics and Statistics, University of Strathclyde, Glasgow G1 1XH, U.K.
}

\begin{abstract}
We present a stochastic age-dependent population model that accounts for Markovian switching and variable delay. By using the approximate value at the nearest grid-point on the left of the delayed argument to estimate the delay function, we propose a class of split-step $\theta$-method for solving stochastic delay age-dependent population equations (SDAPEs) with Markovian switching. We show that the numerical method is convergent under the given conditions. Numerical examples are provided to illustrate our results.
\end{abstract}

Keywords: Stochastic delay age-dependent population equations, Split-step $\theta$-method, Strong convergence, Markovian swtiching, Itô foumula.

\section{Introduction}

Stochastic differential equations (SDEs) are becoming increasingly used to model real world phenomena in different fields, such as economics, biology, finance and population dynamics. As an important branch of SDEs, stochastic population equations have received a great deal of attention. In the present investigation, the random behavior described by different stochastic processes such as Markovian switching, Poisson jumps, and fractional Brownian motion is incorporated into the stochastic age-dependent population equations (SAPEs) (see e.g., [10, 11, 18, 19, 22, 28]). In these population dynamics, one assumes that the system is governed by a principle of causality, that is the future state of the system is determined solely by the present states. However, in real world population system, it takes certain time for individuals to mature as well as for infectious diseases to be cured. This motivates us to develop a more realistic model including some of the past states of the system called time delay to describe the relationship between the causes and their effects (see e.g., [9, 12, 13, 14, 16]). Then this stochastic delay age-dependent population

\footnotetext{
${ }^{*}$ Corresponding author

Email address: wyfei@ahpu.edu.cn (Weiyin Fei )
} 
system with Markovian switching can be defined by the following form:

$$
\left\{\begin{aligned}
d_{t} P_{t}= & {\left[-\frac{\partial P_{t}}{\partial a}-\mu(t, a) P_{t}+f\left(r(t), P_{t}, P_{\delta(t)}\right)\right] d t } & & \\
& +g\left(r(t), P_{t}, P_{\delta(t)}\right) d W_{t}, & & (t, a) \in[0, T] \times[0, A] \\
P(t, a)= & \varphi(t, a), & & (t, a) \in[-\tau, 0] \times[0, A] \\
P(t, 0)= & \int_{0}^{A} \beta(t, a) P(t, a) d a, & & t \in[0, T]
\end{aligned}\right.
$$

where $\tau>0, d_{t} P_{t}=\frac{\partial P_{t}}{\partial t} d t, \delta(t)$ stands for the time delay $(-\tau \leq \delta(t) \leq t), A$ denotes the maximum age, $r(t)$ is a Markov chain, $W_{t}$ is a Wiener process, $P_{t}=P(t, a)$ denotes the population density of age $a$ at time $t, P_{\delta(t)}=P(\delta(t), a)$ denotes the population density of age $a$ at time $\delta(t), \beta(t, a)$ 5 denotes the fertility rate of females of age $a$ at time $t, \mu(t, a)$ denotes the mortality rate of age $a$ at time $t, f\left(r(t), P_{t}, P_{\delta(t)}\right)$ denotes the effects of an external environment for the population system, $g\left(r(t), P_{t}, P_{\delta(t)}\right)$ is a diffusion coefficient.

With its simple algebraic structure, cheap computational cost and acceptable convergence rate, Euler-Maruyama (EM) method has been widely used to solve SDEs (see e.g., 4, 5, 15, 25,

10 27]). Since most SAPEs cannot be solved explicitly, the constructions of efficient computational method have become essential. For example, Zhang et al. [11, 28, 29] investigated the convergence of numerical solutions to SAPEs, Li et al. [10] considered the convergence of numerical solutions to SAPEs with Markovian switching. In recent years, influenced by Higham, Mao and Stuart [6, 7], split-step $\theta$-method (SS $\theta$ method) has attracted a lot of concern due to their

15 advantages in dealing with the flexibility and the stability for the SDEs (see e.g., [2, 16, 23]). Researchers find that the $\mathrm{SS} \theta$ method or its improved forms have desirable stability properties, convergence rates and structure-preserving properties (see e.g., [8, 17]). Tan et al. [22] constructed a class of $\operatorname{SS} \theta$ method for SAPEs with Poisson jumps. In [19], Rathinasamy introduced a class of SS $\theta$ method for SAPEs with Markovian switching. In our stochastic population model (1.1),

20 we allow the time delay to be a function of time, namely variable delay, which is more general than the constant delay. The main difficulty in dealing with variable delays by numerical method is that at the current time-step the delayed argument may not hit a precious time-step (see [16]). In order to overcome this difficulty we use the the approximate value at the nearest grid-point on the left of the delayed argument to estimate the delay function. We then present a class of SS $\theta$

25 method for SDAPEs with Markovian switching. The SS $\theta$ method includes the split-step forward method and the split-step backward Euler method by choosing $\theta=0$ and $\theta=1$ and it is more general than the two methods. The convergent result is proved under the given conditions.

The outline of the paper is organized as follows. In Section 2, we will introduce some basic preliminaries. A class of SS $\theta$ method for solving SDAPEs with Markovian switching will be

30 proposed in Section 3. In Section 4, the $\operatorname{SS} \theta$ method converge strongly to the exact solutions of SDAPEs with Markovian switching will be shown. Numerical experiments will be given in the final section.

\section{Preliminaries}

Throughout this paper, unless otherwise specified, let $(\Omega, \mathcal{F}, \mathbb{P})$ be a complete probability space with a filtration $\left\{\mathcal{F}_{t}\right\}_{t \geq 0}$ satisfying the usual conditions(i.e., it is increasing and right continuous while $\mathcal{F}_{0}$ contains all $\mathbb{P}$-null sets). Let $r(t), t \geq 0$, be a right-continuous Markov chain 
on the probability space taking values in a finite state space $\mathbb{S}=\{1,2, \cdots, N\}$ with generator $\Gamma=\left(\gamma_{i j}\right)_{N \times N}$ given by

$$
\mathbb{P}\{r(t+\Delta)=j \mid r(t)=i\}= \begin{cases}\gamma_{i j} \Delta+o(\Delta) & \text { if } i \neq j, \\ 1+\gamma_{i i} \Delta+o(\Delta) & \text { if } i=j,\end{cases}
$$

where $\Delta>0$. Here $\gamma_{i j} \geq 0$ is the transition rate from $i$ to $j$ if $i \neq j$, while $\gamma_{i i}=-\sum_{j \neq i} \gamma_{i j}$. 35 We assume that the Markov chain $r(\cdot)$ is independent of the Brownian motion. It is well known that almost every sample path of $r(t)$ is a right-continuous step function with a finite number of simple jumps in any finite subinterval of $\mathbb{R}^{+}$.

Let

$$
V=H^{1}([0, A]) \equiv\left\{\varphi \mid \varphi \in L^{2}([0, A]), \frac{\partial \varphi}{\partial x_{i}} \in L^{2}([0, A])\right\}
$$

where $\frac{\partial \varphi}{\partial x_{i}}$ is the generalized partial derivative and $V$ is a Sobolev space. $H=L^{2}([0, A])$ such that 40 $V \hookrightarrow H \equiv H^{\prime} \hookrightarrow V^{\prime}$. $V^{\prime}$ is the dual space of $V$. We denote by $\|\cdot\|,|\cdot|$ and $\|\cdot\|_{*}$ the norms in $V, H$ and $V^{\prime}$ respectively; by $\langle\cdot, \cdot\rangle$ the duality product between $V$ and $V^{\prime}$, and by $(\cdot, \cdot)$ the scalar product in $H$. Let $W_{t}$ be a Wiener process defined on $\left(\Omega, \mathcal{F},\left\{\mathcal{F}_{t}\right\}_{t \geq 0}, \mathbb{P}\right)$ and taking its values in the separable Hilbert space $K$, with increment covariance operator $W$. For an operator $B \in \mathcal{L}(K, H)$ being the space of all bounded linear operators from $K$ to $H$, we denote by $\|B\|_{2}$ be the Hilbert-

45 Schmidt norm, i.e. $\|B\|_{2}=\operatorname{trace}\left(B W B^{T}\right)$. Let $\left.C=C([-\tau, t]) ; H\right)$ be the space of all continuous function from $[-\tau, t]$ into $H$, with sup-norm $\left\|\phi_{t}\right\|_{C}=\sup _{-\tau \leq s \leq t}|\phi(s)|$, and $\left.L_{H}^{p}=L^{p}([0, T]) ; H\right)$.

The integral version of 1.1 is given by the equations:

$$
\left\{\begin{array}{rlrl}
P_{t}= & P_{0}-\int_{0}^{t} \frac{\partial P_{s}}{\partial a} d s-\int_{0}^{t} \mu(s, a) P_{s} d s+\int_{0}^{t} f\left(r(s), P_{s}, P_{\delta(s)}\right) d s & \\
& +\int_{0}^{t} g\left(r(s), P_{s}, P_{\delta(s)}\right) d W_{s}, & & (t, a) \in[0, T] \times[0, A] \\
P(t, a)= & \varphi(t, a), & (t, a) \in[-\tau, 0] \times[0, A] \\
P(t, 0)= & \int_{0}^{A} \beta(t, a) P(t, a) d a, & t \in[0, T]
\end{array}\right.
$$

where $f(i, \cdot, \cdot): \mathbb{S} \times L_{H}^{2} \times L_{H}^{2} \rightarrow H$ be a family of non-linear operators, $\mathcal{F}_{t}$ measurable almost surely in $t, g(i, \cdot, \cdot): \mathbb{S} \times L_{H}^{2} \times L_{H}^{2} \rightarrow \mathcal{L}(K, H)$ is the family of non-linear operators, $\mathcal{F}_{t}$ measurable almost surely in $t$, the other notions are defined in 1.1 . As the standing hypotheses we always 50 assume that:

- The Lipschitz continuous function $\delta:[0,+\infty) \rightarrow \mathbb{R}$ satisfies

$$
-\tau \leq \delta(t) \leq t \quad \text { and } \quad|\delta(t)-\delta(s)| \leq \rho|t-s|, \quad \forall t, s \geq 0
$$

for a positive constant $\rho$.

- There exist a constant $\bar{K}>0$ such that for $-\tau \leq s<t \leq 0,0 \leq a \leq A$

$$
\mathbb{E}\left[\sup _{-\tau \leq s<t \leq 0}|\varphi(t, a)-\varphi(s, a)|^{2}\right] \leq \bar{K}|t-s| \text {. }
$$


- For $k \geq 2$ and $0 \leq a \leq A$,

$$
\mathbb{E}\left[\sup _{-\tau \leq t \leq 0}|\varphi(t, a)|^{k}\right]<\infty
$$

Moreover, we shall impose the following assumptions:

$(\mathbb{H} 11) f(i, 0,0)=0, g(i, 0,0)=0, \forall i \in S$.

(H12) There exists a positive constant $L$ such that for $x, y \in C, i \in \mathbb{S}$,

$$
|f(i, x, y)-f(i, \bar{x}, \bar{y})| \vee\|g(i, x, y)-g(i, \bar{x}, \bar{y})\|_{2} \leq L\left(\|x-\bar{x}\|_{C}+\|y-\bar{y}\|_{C}\right) .
$$

$(\mathbb{H} 3) \mu(t, a), \beta(t, a)$ are continuous in $[0, T] \times[0, A]$ and there exist positive constants $\mu_{0}, \bar{\alpha}$ and $\bar{\beta}$ such that

$$
\begin{aligned}
& 0 \leq \mu_{0} \leq \mu(t, a) \leq \bar{\alpha}<\infty, \\
& 0 \leq \beta(t, a) \leq \bar{\beta}<\infty .
\end{aligned}
$$

From $(\mathbb{H} 1)$ and $(\mathbb{H} 2)$, we obtain that for $x, y \in C$

$$
\begin{aligned}
& |f(i, x, y)|^{2}=|f(i, x, y)-f(i, 0,0)|^{2} \leq 2 L^{2}\left(\|x\|_{C}^{2}+\|y\|_{C}^{2}\right), \\
& \|g(i, x, y)\|_{2}^{2}=\|g(i, x, y)-g(i, 0,0)\|_{2}^{2} \leq 2 L^{2}\left(\|x\|_{C}^{2}+\|y\|_{C}^{2}\right) .
\end{aligned}
$$

These inequalitis will be very useful in what follows.

\section{Split-step $\theta$-method}

For any given time $T>0$, there exist sufficiently large positive integers $m$ and $M$, such that

$$
0<\Delta=\frac{\tau}{m}=\frac{T}{M}<1 .
$$

Let $t_{n}=n \Delta, \Delta W_{n}=W_{t_{n+1}}-W_{t_{n}}$. We denote $\lfloor u\rfloor$ by the integer part of the real number $u$. For $t \in[0, T]$, there is an integer $n \in[0, M-1]$ such that $t \in[n \Delta,(n+1) \Delta)$. Recalling 2.2$]$ that $-\tau \leq \delta(t) \leq t$, we obtain

$$
-m \Delta=-\tau \leq \delta(n \Delta) \leq n \Delta .
$$

Thus, we get

$$
-m \leq \delta(n \Delta) / \Delta \leq n,
$$

which means

$$
-m \leq\lfloor\delta(n \Delta) / \Delta\rfloor \leq n .
$$

Hence, we obtain

$$
-\tau=-m \Delta \leq\lfloor\delta(n \Delta) / \Delta\rfloor \Delta \leq n \Delta .
$$


Then the $\operatorname{SS} \theta$ method is defined by the following:

$$
\left\{\begin{aligned}
Q_{n}^{*}= & Q_{n}-\frac{\partial Q_{n}}{\partial a} \Delta+(1-\theta)\left[-\mu\left(t_{n}, a\right) Q_{n}+f\left(r_{n}^{\Delta}, Q_{n}, Q_{\lfloor\delta(n \Delta) / \Delta\rfloor}\right)\right] \Delta, & & \\
& +\theta\left[-\mu\left(t_{n}, a\right) Q_{n}^{*}+f\left(r_{n}^{\Delta}, Q_{n}^{*}, Q_{\lfloor\delta(n \Delta) / \Delta\rfloor}\right)\right] \Delta, & & 0 \leq n \leq M-1 \\
Q_{n+1}= & Q_{n}^{*}+g\left(r_{n}^{\Delta}, Q_{n}^{*}, Q_{\lfloor\delta(n \Delta) / \Delta\rfloor}\right) \Delta W_{n}, & & 0 \leq n \leq M-1 \\
Q_{n} & =\varphi\left(t_{n}, a\right) . & & -m \leq n \leq 0
\end{aligned}\right.
$$

with the initial value $Q_{0}=\varphi(0, a), r_{0}^{\Delta}=r_{0}, Q_{n}(t, 0)=\int_{0}^{A} \beta(t, a) Q_{n} d a, r_{n}^{\Delta}=r(n \Delta)$, for $0 \leq n \leq$ $M-1$. Let $Q_{n}$ be the approximation to $P\left(t_{n}, a\right)$ for $t_{n}=n \Delta$. We set $0 \leq \theta \leq 1$. The choice $\theta=1$ gives the split-step backward Euler method. If $\theta=0$, the $\operatorname{SS} \theta$ method degenerates to the split-step forward method. The first equation in (3.1) is an implicit equation in $Q_{n}^{*}$ that must be 60 $\quad$ solved in order to obtain the intermediate approximation $Q_{n}^{*}$. Having obtained $Q_{n}^{*}$, substituting it into the second of 3.1 produces the next approximation $Q_{n+1}$. Using the classical Banach contraction mapping theorem, the first equation in (3.1) has a unique fixed $Q_{n}^{*}$ (see [21]). We now present present the following lemma.

Lemma 3.1. Assume that $f: \mathbb{R} \times \mathbb{R} \times[0, T] \longmapsto \mathbb{R}$ satisfies ( $\mathbb{H} 1)$ and $(\mathbb{H} 2)$. Let $0<\theta \leq 1$ and ${ }_{65} 0<\Delta<1 /(L \theta)$. Then for any $a, b, c \in \mathbb{R}$, the implicit equation $x=a+\Delta \theta f(b, x, c)$ has a unique solution $x$.

We define the following step functions:

$$
\begin{aligned}
\bar{Z}_{t} & =\sum_{n=0}^{M-1} Q_{n} \mathbb{I}_{[n \Delta,(n+1) \Delta)}(t), \\
\widetilde{Z}_{t} & =\sum_{n=0}^{M-1} Q_{[\delta(n \Delta) / \Delta]} \mathbb{I}_{[n \Delta,(n+1) \Delta)}(t), \\
\hat{Z}_{t} & =\sum_{n=0}^{M-1} Q_{n}^{*} \mathbb{I}_{[n \Delta,(n+1) \Delta)}(t), \\
\bar{r}(t) & =\sum_{n=0}^{M-1} r_{n}^{\Delta} \mathbb{I}_{[n \Delta,(n+1) \Delta)}(t),
\end{aligned}
$$

where $\mathbb{I}$ is the indicator function. It is convenient to use the continuous-time approximation

$$
\left\{\begin{array}{rlrl}
Q_{t}= & P_{0}-\int_{0}^{t} \frac{\partial Q_{n}}{\partial a} d s+\int_{0}^{t}(1-\theta)\left[-\mu(s, a) \bar{Z}_{s}+f\left(\bar{r}(s), \bar{Z}_{s}, \widetilde{Z}_{s}\right)\right] d s & \\
& +\int_{0}^{t} \theta\left[-\mu(s, a) \hat{Z}_{s}+f\left(\bar{r}(s), \hat{Z}_{s}, \widetilde{Z}_{s}\right)\right] d s+\int_{0}^{t} g\left(\bar{r}(s), \hat{Z}_{s}, \widetilde{Z}_{s}\right) d W_{s}, & & 0 \leq t \leq T \\
Q_{t}= & \varphi(t, a), & & -\tau \leq t \leq 0
\end{array}\right.
$$

with $Q_{0}=P_{0}=\varphi(0, a), Q(t, 0)=\int_{0}^{A} \beta(t, a) Q_{t} d a, Q_{t}=Q(t, a), \bar{r}(0)=i_{0}$. Clearly, 3.3) can also be written as

$$
Q_{t}=Q_{n}-\frac{\partial Q_{n}}{\partial a}\left(t-t_{n}\right)+(1-\theta)\left[-\mu\left(t_{n}, a\right) Q_{n}+f\left(r_{n}^{\Delta}, Q_{n}, Q_{[\delta(n \Delta) / \Delta)}\right)\right]\left(t-t_{n}\right)
$$




$$
\begin{aligned}
& +\theta\left[-\mu\left(t_{n}, a\right) Q_{n}^{*}+f\left(r_{n}^{\Delta}, Q_{n}^{*}, Q_{\lfloor\delta(n \Delta) / \Delta\rfloor}\right)\right]\left(t-t_{n}\right) \\
& +g\left(r_{n}^{\Delta}, Q_{n}^{*}, Q_{\lfloor\delta(n \Delta) / \Delta\rfloor}\right)\left(W_{t}-W_{t_{n}}\right),
\end{aligned}
$$

for $t_{n} \leq t<t_{n+1}$. It is straightforward to check that $\bar{Z}\left(t_{n}, a\right)=Q_{n}=Q\left(t_{n}, a\right)$, for $-m \leq n \leq M-1$ and $P_{t}=Q_{t}=\varphi(t, a)$, for $-\tau \leq t \leq 0$.

\section{Strong convergence}

In this section, We shall discuss the strong convergence of $\operatorname{SS} \theta$ approximate solution under the given conditions. We first give several lemmas which are useful for the main results.

Lemma 4.1. Let $P_{t}$ be the solution of 2.1). Then

$$
-\left\langle P_{t}, \frac{\partial P_{t}}{\partial a}\right\rangle \leq \frac{1}{2} A \bar{\beta}^{2}\left|P_{t}\right|^{2}
$$

where $A, \bar{\beta}$ is the same as before.

Proof. It is easy to see that

$$
\begin{aligned}
-\left\langle P_{t}, \frac{\partial P_{t}}{\partial a}\right\rangle & =-\int_{0}^{A} P_{t} \frac{\partial P_{t}}{\partial a} d a=-\int_{0}^{A} P(t, a) d_{a} P(t, a) \\
& =-\frac{1}{2} \int_{0}^{A} d_{a} P^{2}(t, a)=-\frac{1}{2}\left(P^{2}(t, A)-P^{2}(t, 0)\right) .
\end{aligned}
$$

Recalling that $P(t, a)$ denote the population density of the age $a$ at time $t$, we have $P(t, A)=0$ for the maximum age A and any $t \in[0, T]$. By (2.1) and the Cauchy-Schwarz inequality, we have

$$
\begin{aligned}
-\left\langle P_{t}, \frac{\partial P_{t}}{\partial a}\right\rangle & =\frac{1}{2} P^{2}(t, 0)=\frac{1}{2}\left(\int_{0}^{A} \beta(t, a) P(t, a) d a\right)^{2} \\
& \leq \frac{1}{2} \int_{0}^{A} \beta^{2}(t, a) d a \int_{0}^{A} P^{2}(t, a) d a \leq \frac{1}{2} A \bar{\beta}^{2}\left|P_{t}\right|^{2} .
\end{aligned}
$$

Thus, the proof is complete.

Remark 4.2. If we denote $f_{0}\left(P_{t}\right):=-\frac{\partial P_{t}}{\partial a}-\mu(t, a) P_{t}$ for all $P_{t} \in V$. By Lemma 4.1 we have

$$
\left\langle f_{0}\left(P_{t}\right), P_{t}\right\rangle=\left\langle-\frac{\partial P_{t}}{\partial a}-\mu(t, a) P_{t}, P_{t}\right\rangle \leq\left(\frac{1}{2} A \bar{\beta}^{2}-\mu_{0}\right)\left|P_{t}\right|^{2}, \quad \forall P_{t} \in V
$$

which means that $f_{0}$ is a linear operator in $V$ and satisfies the one-sided linear growth condition.

75 This viewpoint will be of great help in the following analysis.

Lemma 4.3. Under the assumptions ( $\mathbb{H} 1)-(\mathbb{H} 3)$, for any $k \geq 2$, there is a constants $C_{1}>0$ such that

$$
\mathbb{E}\left[\sup _{-\tau \leq t \leq T}\left|P_{t \wedge \tau_{R}}\right|^{k}\right] \leq C_{1},
$$

where $\tau_{R}=\inf \left\{t \geq 0:\left|P_{t}\right| \geq R\right\}$ and $C_{1}$ is independent of $\Delta$. 
Proof. Applying the Itô formula to $\left|P_{t \wedge \tau_{R}}\right|^{k}$ yields

$$
\begin{aligned}
\left|P_{t}\right|^{k}= & \left|P_{0}\right|^{k}+k \int_{0}^{t \wedge \tau_{R}}\left|P_{s}\right|^{k-2}\left\langle-\frac{\partial P_{s}}{\partial a}, P_{s}\right\rangle d s+k \int_{0}^{t \wedge \tau_{R}}\left|P_{s}\right|^{k-2}\left\langle-\mu(s, a) P_{s}, P_{\delta(s)}\right\rangle d s \\
& +k \int_{0}^{t \wedge \tau_{R}}\left|P_{s}\right|^{k-2}\left(P_{s}, f\left(r(s), P_{s}, P_{\delta(s)}\right)\right) d s+\frac{k}{2} \int_{0}^{t \wedge \tau_{R}}\left|P_{s}\right|^{k-2}\left\|g\left(r(s), P_{s}, P_{\delta(s)}\right)\right\|_{2}^{2} d s \\
& +\frac{k(k-2)}{2} \int_{0}^{t \wedge \tau_{R}}\left|P_{s}\right|^{k-4}\left\|\left(P_{s}, g\left(r(s), P_{s}, P_{\delta(s)}\right)\right)\right\|_{2}^{2} d s+M\left(t \wedge \tau_{R}\right),
\end{aligned}
$$

where

$$
M\left(t \wedge \tau_{R}\right)=k \int_{0}^{t \wedge \tau_{R}}\left|P_{s}\right|^{k-2}\left(P_{s}, g\left(r(s), P_{s}, P_{\delta(s)}\right)\right) d W_{s} .
$$

By Lemma 4.1, we have

$$
\left\langle-\frac{\partial P_{s}}{\partial a}, P_{s}\right\rangle \leq \frac{1}{2} A \bar{\beta}^{2}\left|P_{s}\right|^{2}
$$

By the assumptions ( $\mathbb{H} 1)-(\mathbb{H} 3)$, we get that

$$
\begin{gathered}
k \int_{0}^{t \wedge \tau_{R}}\left|P_{s}\right|^{k-2}\left\langle-\mu(s, a) P_{s}, P_{\delta(s)}\right\rangle d s \leq-k \mu_{0} \int_{0}^{t \wedge \tau_{R}} \sup _{-\tau \leq u \leq s}\left|P_{u}\right|^{k} d s, \\
k \int_{0}^{t \wedge \tau_{R}}\left|P_{s}\right|^{k-2}\left(P_{s}, f\left(r(s), P_{s}, P_{\delta(s)}\right)\right) d s \leq 2 k L \int_{0}^{t \wedge \tau_{R}} \sup _{-\tau \leq u \leq s}\left|P_{u}\right|^{k} d s, \\
\frac{k}{2} \int_{0}^{t \wedge \tau_{R}}\left|P_{s}\right|^{k-2}\left\|g\left(r(s), P_{s}, P_{\delta(s)}\right)\right\|_{2}^{2} d s \leq k L^{2} \int_{0}^{t \wedge \tau_{R}}\left|P_{s}\right|^{k-2}\left(\left\|P_{s}\right\|_{C}^{2}+\left\|P_{\delta(s)}\right\|_{C}^{2}\right) d s \\
\leq 2 k L^{2} \int_{0}^{t \wedge \tau_{R}} \sup _{-\tau \leq u \leq s}\left|P_{u}\right|^{k} d s, \\
\frac{k(k-2)}{2} \int_{0}^{t \wedge \tau_{R}}\left|P_{s}\right|^{k-4}\left\|\left(P_{s}, g\left(r(s), P_{s}, P_{\delta(s)}\right)\right)\right\|_{2}^{2} d s \leq 2 k(k-2) L^{2} \int_{0}^{t \wedge \tau_{R}} \sup _{-\tau \leq u \leq s}\left|P_{u}\right|^{k} d s .
\end{gathered}
$$

Substituting (4.3)-(4.7) into (4.2) yields

$$
\left|P_{t}\right|^{k} \leq\left|P_{0}\right|^{k}+\frac{k}{2}\left(A \bar{\beta}^{2}-2 \mu_{0}+4(k-2) L^{2}+4 L\right) \int_{0}^{t \wedge \tau_{R}} \sup _{-\tau \leq u \leq s}\left|P_{u}\right|^{k} d s+M\left(t \wedge \tau_{R}\right) .
$$

Note that for $t \in[0, T]$,

$$
\mathbb{E}\left[\sup _{-\tau \leq u \leq t}\left|P_{u \wedge \tau_{R}}\right|^{k}\right]=\mathbb{E}\left[\sup _{-\tau \leq u \leq 0}\left|P_{u \wedge \tau_{R}}\right|^{k}\right] \vee \mathbb{E}\left[\sup _{0 \leq u \leq t}\left|P_{u \wedge \tau_{R}}\right|^{k}\right]
$$


Now, it follows that

$$
\begin{aligned}
\mathbb{E}\left[\sup _{-\tau \leq u \leq t}\left|P_{u}\right|^{k}\right] & \leq \mathbb{E}\left[\sup _{-\tau \leq u \leq 0}|\varphi(u, a)|^{k}\right] \\
& +\frac{k}{2}\left(A \bar{\beta}^{2}-2 \mu_{0}+4(k-2) L^{2}+4 L\right) \int_{0}^{t \wedge \tau_{R}} E\left[\sup _{-\tau \leq u \leq s}\left|P_{u}\right|^{k}\right] d s \\
& +k \mathbb{E}\left[\sup _{0 \leq u \leq t} \int_{0}^{t \wedge \tau_{R}}\left|P_{s}\right|^{k-2}\left(P_{s}, g\left(r(s), P_{s}, P_{\delta(s)}\right) d W_{s}\right)\right] .
\end{aligned}
$$

By the Burkholder-Davis-Gundy inequality, we have

$$
\begin{aligned}
& \mathbb{E}\left[\sup _{0 \leq s \leq t} \int_{0}^{t \wedge \tau_{R}}\left|P_{s}\right|^{k-2}\left(P_{s}, g\left(r(s), P_{s}, P_{\delta(s)}\right)\right) d W_{s}\right] \\
& \leq 3 \mathbb{E}\left[\left.\left.\int_{0}^{t \wedge \tau_{R}}|| P_{u}\right|^{k-2}\left(P_{s}, g\left(r(s), P_{s}, P_{\delta(s)}\right)\right)\right|^{2} d s\right]^{1 / 2} \\
& \leq 3 \mathbb{E}\left[\sup _{-\tau \leq u \leq t}\left|P_{u}\right|^{k / 2}\left[\int_{0}^{t \wedge \tau_{R}}\left|P_{s}\right|^{k-2}\left\|g\left(r(s), P_{s}, P_{\delta(s)}\right)\right\|_{2}^{2} d s\right]^{1 / 2}\right] \\
& \leq \frac{1}{2 k} \mathbb{E}\left[\sup _{-\tau \leq u \leq t}\left|P_{u}\right|^{k}\right]+K_{0} \mathbb{E}\left[\int_{0}^{t \wedge \tau_{R}}\left|P_{s}\right|^{k-2}\left\|g\left(r(s), P_{s}, P_{\delta(s)}\right)\right\|_{2}^{2} d s\right] \\
& \leq \frac{1}{2 k} \mathbb{E}\left[\sup _{-\tau \leq u \leq t}\left|P_{u}\right|^{k}\right]+4 K_{0} L^{2} \int_{0}^{t \wedge \tau_{R}} \mathbb{E}\left[\sup _{-\tau \leq u \leq s}\left|P_{s}\right|^{k}\right] d s
\end{aligned}
$$

for a positive constant $K_{0}$. Note that

$$
\begin{aligned}
\mathbb{E}\left[\left|P_{s}\right|^{k-2}\left\|g\left(r(s), P_{s}, P_{\delta(s)}\right)\right\|_{2}^{2}\right] & \leq 2 L^{2} \mathbb{E}\left[\left|P_{s}\right|^{k-2}\left(\left\|P_{s}\right\|_{C}^{2}+\left\|P_{\delta(s)}\right\|_{C}^{2}\right)\right] \\
& \leq 4 L^{2} \mathbb{E}\left[\sup _{-\tau \leq u \leq t}\left|P_{u}\right|^{k}\right] .
\end{aligned}
$$

Consequently,

$$
\begin{aligned}
\mathbb{E}\left[\sup _{-\tau \leq u \leq t}\left|P_{u}\right|^{k}\right] & \leq \mathbb{E}\left[\sup _{-\tau \leq u \leq 0}|\varphi(u, a)|^{k}\right]+\frac{1}{2} \mathbb{E}\left[\sup _{-\tau \leq u \leq t}\left|P_{u}\right|^{k}\right] \\
& +\frac{k}{2}\left(A \bar{\beta}^{2}-2 \mu_{0}+4(k-2) L^{2}+4 L+8 K_{0} L^{2}\right) \int_{0}^{t \wedge \tau_{R}} \mathbb{E}\left[\sup _{-\tau \leq u \leq s}\left|P_{u}\right|^{k}\right] d s .
\end{aligned}
$$

Using the Gronwall inequality implies the required result with

$$
C_{1}=2 \mathbb{E}\left[\sup _{-\tau \leq u \leq 0}|\varphi(u, a)|^{k}\right] e^{k\left(A \bar{\beta}^{2}-2 \mu_{0}+4(k-2) L^{2}+4 L+8 K_{0} L^{2}\right) T / 2} .
$$

Thus, the proof is complete.

Lemma 4.4. Under the assumptions $(\mathbb{H} 1)-(\mathbb{H} 3)$, let $\Delta<\min \left\{1,1 /\left(\sqrt{12\left(\bar{\alpha}^{2}+2 L^{2}\right)}\right)\right\}$ and $\mathbb{E}\left|\frac{\partial Q_{n}}{\partial a}\right|^{2}<$ $\infty, 0 \leq n \leq M-1$. Then there exist positive constants $C_{2}, C_{3}, C_{4}$, such that

$$
\mathbb{E}\left|Q_{n}^{*}\right|^{2} \leq C_{2} \mathbb{E}\left|Q_{n}\right|^{2}+C_{3} \mathbb{E}\left|Q_{\lfloor\delta(n \Delta) / \Delta\rfloor}\right|^{2}+C_{4}, \quad 0 \leq n \leq M-1
$$

where $Q_{n}, Q_{n}^{*}$ is defined in (3.1) and $C_{2}, C_{3}, C_{4}$ is independent of $\Delta$. 
Proof. Squaring both sides of the first equation in 3.1], we have

$$
\begin{aligned}
\left|Q_{n}^{*}\right|^{2} \leq & 3\left|Q_{n}\right|^{2}+3\left|\frac{\partial Q_{n}}{\partial a}\right|^{2} \Delta^{2}+3 \mid(1-\theta)\left[-\mu\left(t_{n}, a\right) Q_{n}+f\left(r_{n}^{\Delta}, Q_{n}, Q_{\lfloor\delta(n \Delta) / \Delta\rfloor}\right)\right] \\
& +\left.\theta\left[-\mu\left(t_{n}, a\right) Q_{n}^{*}+f\left(r_{n}^{\Delta}, Q_{n}^{*}, Q_{\lfloor\delta(n \Delta) / \Delta\rfloor}\right)\right]\right|^{2} \Delta^{2} \\
\leq & 3\left|Q_{n}\right|^{2}+3\left|\frac{\partial Q_{n}}{\partial a}\right|^{2} \Delta^{2}+12\left(\bar{\alpha}^{2}\left|Q_{n}\right|^{2}+\left|f\left(r_{n}^{\Delta}, Q_{n}, Q_{\lfloor\delta(n \Delta) / \Delta\rfloor}\right)\right|^{2}\right) \Delta^{2} \\
& +12\left(\bar{\alpha}^{2}\left|Q_{n}^{*}\right|^{2}+\mid f\left(r_{n}^{\Delta}, Q_{n}^{*},\left.Q_{\lfloor\delta(n \Delta) / \Delta\rfloor}\right|^{2}\right) \Delta^{2} .\right.
\end{aligned}
$$

By the assumptions ( $\mathbb{H} 1)-(\mathbb{H} 3)$, we have

$$
\begin{aligned}
\left|Q_{n}^{*}\right|^{2} \leq & 3\left|Q_{n}\right|^{2}+3\left|\frac{\partial Q_{n}}{\partial a}\right|^{2} \Delta^{2}+12\left[\bar{\alpha}^{2}\left|Q_{n}\right|^{2}+2 L^{2}\left|Q_{n}\right|^{2}+2 L^{2}\left|Q_{\lfloor\delta(n \Delta) / \Delta\rfloor}\right|^{2}\right] \Delta^{2} \\
& \left.+12\left[\bar{\alpha}^{2}\left|Q_{n}^{*}\right|^{2}+2 L^{2}\left|Q_{n}^{*}\right|^{2}+2 L^{2}\left|Q_{\lfloor\delta(n \Delta) / \Delta\rfloor}\right|^{2}\right)\right] \Delta^{2} \\
= & {\left[3+12\left(\bar{\alpha}^{2}+2 L^{2}\right) \Delta^{2}\right]\left|Q_{n}\right|^{2}+12\left(\bar{\alpha}^{2}+2 L^{2}\right) \Delta^{2}\left|Q_{n}^{*}\right|^{2} } \\
& +3\left|\frac{\partial Q_{n}}{\partial a}\right|^{2} \Delta^{2}+48 L^{2} \Delta^{2}\left|Q_{\lfloor(n \Delta) / \Delta\rfloor}\right|^{2}
\end{aligned}
$$

Hence, we get

$$
\begin{aligned}
\mathbb{E}\left|Q_{n}^{*}\right|^{2} \leq & \frac{\left(3+12\left(\bar{\alpha}^{2}+2 L^{2}\right) \Delta^{2}\right.}{1-12\left(\bar{\alpha}^{2}+2 L^{2}\right) \Delta^{2}} \mathbb{E}\left|Q_{n}\right|^{2}+\frac{48 L^{2} \Delta^{2}}{1-12\left(\bar{\alpha}^{2}+2 L^{2}\right) \Delta^{2}} \mathbb{E}\left|Q_{\lfloor\delta(n \Delta) / \Delta\rfloor}\right|^{2} \\
& +\frac{3 \mathbb{E}\left|\frac{\partial Q_{n}}{\partial a}\right|^{2} \Delta^{2}}{1-12\left(\bar{\alpha}^{2}+2 L^{2}\right) \Delta^{2}} \\
\leq & C_{2} \mathbb{E}\left|Q_{n}\right|^{2}+C_{3} \mathbb{E}\left|Q_{\lfloor\delta(n \Delta) / \Delta\rfloor}\right|^{2}+C_{4} .
\end{aligned}
$$

so Thus, the proof is complete.

Lemma 4.5. Under the assumptions $(\mathbb{H} 1)-(\mathbb{H} 3)$ and let $\Delta<\min \left\{1,1 /\left(\sqrt{12\left(\bar{\alpha}^{2}+2 L^{2}\right)}\right)\right\}$, there exist a positive constant $C_{5}$ independent of $\Delta$ such that

where $\sigma_{R}=\inf \left\{t \geq 0:\left|Q_{t}\right| \geq R\right\}$.

$$
\mathbb{E}\left[\sup _{-\tau \leq t \leq T}\left|Q_{t \wedge \sigma_{R}}\right|^{2}\right] \leq C_{5},
$$

Proof: Applying the Itô formula to $\left|Q_{t \wedge \sigma_{R}}\right|^{2}$ yields

$$
\begin{aligned}
\left|Q_{t \wedge \sigma_{R}}\right|^{2}= & \left|Q_{0}\right|^{2}+2 \int_{0}^{t \wedge \sigma_{R}}\left\langle-\frac{\partial Q_{s}}{\partial a}, Q_{s}\right\rangle d s \\
& +2 \int_{0}^{t \wedge \sigma_{R}}\left((1-\theta) f\left(\bar{r}(s), \bar{Z}_{s}, \widetilde{Z}_{s}\right)+\theta f\left(\bar{r}(s), \hat{Z}_{s}, \widetilde{Z}_{s}\right), Q_{s}\right) d s \\
& -2 \int_{0}^{t \wedge \sigma_{R}}\left(\mu(s, a)\left[(1-\theta) \bar{Z}_{s}+\theta \hat{Z}_{s}\right], Q_{s}\right) d s+\int_{0}^{t \wedge \sigma_{R}}\left\|g\left(\bar{r}(s), \hat{Z}_{s}, \widetilde{Z}_{s}\right)\right\|_{2}^{2} d s \\
& +2 \int_{0}^{t \wedge \sigma_{R}}\left(Q_{s}, g\left(\bar{r}(s), \hat{Z}_{s}, \widetilde{Z}_{s}\right) d W_{s}\right) .
\end{aligned}
$$


Recalling Lemma 4.1, we have

$$
\left\langle-\frac{\partial Q_{s}}{\partial a}, Q_{s}\right\rangle \leq \frac{1}{2} A \bar{\beta}^{2}\left|Q_{s}\right|^{2} .
$$

By the elementary inequality $2 a b \leq a^{2}+b^{2}$ and $(\mathbb{H} 1)-(\mathbb{H} 3)$, we have

$$
\begin{gathered}
2 \int_{0}^{t \wedge \sigma_{R}}\left((1-\theta) f\left(\bar{r}(s), \bar{Z}_{s}, \widetilde{Z}_{s}\right), Q_{s}\right) d s \leq \int_{0}^{t \wedge \sigma_{R}}\left|Q_{s}\right|^{2} d s+2 L^{2} \int_{0}^{t \wedge \sigma_{R}}\left(\left\|\bar{Z}_{s}\right\|_{C}^{2}+\left\|\widetilde{Z}_{s}\right\|_{C}^{2}\right) d s \\
2 \int_{0}^{t \wedge \sigma_{R}}\left((1-\theta) f\left(\bar{r}(s), \hat{Z}_{s}, \widetilde{Z}_{s}\right), Q_{s}\right) d s \leq \int_{0}^{t \wedge \sigma_{R}}\left|Q_{s}\right|^{2} d s+2 L^{2} \int_{0}^{t \wedge \sigma_{R}}\left(\left\|\hat{Z}_{s}\right\|_{C}^{2}+\left\|\widetilde{Z}_{s}\right\|_{C}^{2}\right) d s \\
-2 \int_{0}^{t \wedge \sigma_{R}}\left(\mu(s, a)(1-\theta) \bar{Z}_{s}, Q_{s}\right) d s \leq \bar{\alpha} \int_{0}^{t \wedge \sigma_{R}}\left(\left|\bar{Z}_{s}\right|^{2}+\left|Q_{s}\right|^{2}\right) d s \\
-2 \int_{0}^{t \wedge \sigma_{R}}\left(\theta \mu(s, a) \hat{Z}_{s}, Q_{s}\right) d s \leq \bar{\alpha} \int_{0}^{t \wedge \sigma_{R}}\left(\left|Q_{s}\right|^{2}+\left|\hat{Z}_{s}\right|^{2}\right) d s \\
\quad \int_{0}^{t \wedge \sigma_{R}}\left\|g\left(\bar{r}(s), \hat{Z}_{s}, \widetilde{Z}_{s}\right)\right\|_{2}^{2} d s \leq 2 L^{2} \int_{0}^{t \wedge \sigma_{R}}\left(\left\|\hat{Z}_{s}\right\|_{C}^{2}+\left\|\widetilde{Z}_{s}\right\|_{C}^{2}\right) d s
\end{gathered}
$$

Inserting (4.11)-(4.16) into 4.10) gives

$$
\begin{aligned}
\left|Q_{t \wedge \sigma_{R}}\right|^{2} \leq & \left|Q_{0}\right|^{2}+K_{1} \int_{0}^{t \wedge \sigma_{R}}\left|Q_{s}\right|^{2} d s+K_{2} \int_{0}^{t \wedge \sigma_{R}}\left\|\bar{Z}_{s}\right\|_{C}^{2} d s \\
& +K_{3} \int_{0}^{t \wedge \sigma_{R}}\left\|\hat{Z}_{s}\right\|_{C}^{2} d s+K_{4} \int_{0}^{t \wedge \sigma_{R}}\left\|\widetilde{Z}_{s}\right\|_{C}^{2} d s+M_{1}\left(t \wedge \sigma_{R}\right),
\end{aligned}
$$

where

$$
\begin{aligned}
& K_{1}=A^{2} \bar{\beta}^{2}+2 \bar{\alpha}+2, K_{2}=2 L^{2}+\bar{\alpha}, K_{3}=4 L^{2}+\bar{\alpha} \\
& K_{4}=6 L^{2}, M_{1}\left(t \wedge \sigma_{R}\right)=2 \int_{0}^{t \wedge \sigma_{R}}\left(Q_{s}, g\left(\bar{r}(s), \hat{Z}_{s}, \widetilde{Z}_{s}\right) d W_{s}\right)
\end{aligned}
$$

By Lemma 4.4, it follows that for any $t_{1} \in[0, T]$

$$
\begin{aligned}
& \mathbb{E}\left[\sup _{0 \leq t \leq t_{1}}\left|Q_{t \wedge \sigma_{R}}\right|^{2}\right] \\
& \leq \mathbb{E}\left|Q_{0}\right|^{2}+K_{1} \mathbb{E}\left[\sup _{0 \leq t \leq t_{1}} \int_{0}^{t \wedge \sigma_{R}}\left|Q_{s}\right|^{2} d s\right]+\left(K_{2}+K_{3} C_{2}\right) \mathbb{E}\left[\sup _{0 \leq t \leq t_{1}} \int_{0}^{t \wedge \sigma_{R}}\left|\bar{Z}_{s}\right|^{2} d s\right] \\
& \quad+\left(K_{4}+K_{3} C_{3}\right) \mathbb{E}\left[\sup _{0 \leq t \leq t_{1}} \int_{0}^{t \wedge \sigma_{R}}\left|\widetilde{Z}_{s}\right|^{2} d s\right]+K_{2} C_{4} T+2 \mathbb{E}\left[\sup _{0 \leq t \leq t_{1}} M_{1}\left(t \wedge \sigma_{R}\right)\right] .
\end{aligned}
$$

For $s \in[0, T]$, we have

$$
\begin{aligned}
& \mathbb{E}\left\|\bar{Z}_{s}\right\|_{C}^{2} \leq \mathbb{E}\left[\sup _{-\tau \leq u \leq s}\left|Q_{u}\right|^{2}\right], \\
& \mathbb{E}\left\|\widetilde{Z}_{s}\right\|_{C}^{2} \leq \mathbb{E}\left[\sup _{-\tau \leq u \leq s}\left|Q_{u}\right|^{2}\right] .
\end{aligned}
$$


Inserting this into (4.18) gives

$$
\begin{aligned}
& \mathbb{E}\left[\sup _{0 \leq t \leq t_{1}}\left|Q_{t \wedge \sigma_{R}}\right|^{2}\right] \\
& \leq \mathbb{E}\left|Q_{0}\right|^{2}+K_{5} \int_{0}^{t_{1} \wedge \sigma_{R}} \mathbb{E}\left[\sup _{-\tau \leq u \leq s}\left|Q_{u}\right|^{2} d s\right]+K_{2} C_{4} T+2 \mathbb{E}\left[\sup _{0 \leq t \leq t_{1}} M_{1}\left(t \wedge \sigma_{R}\right)\right],
\end{aligned}
$$

where

$$
K_{5}=K_{1}+K_{2}+K_{3} C_{2}+K_{4}+K_{3} C_{3} .
$$

By the Burkholder-Davis-Gundy inequality and the Young inequality, we have

$$
\begin{aligned}
& \mathbb{E}\left[\sup _{0 \leq t \leq t_{1}} \int_{0}^{t \wedge \sigma_{R}}\left(Q_{s}, g\left(\bar{r}(s), \hat{Z}_{s}, \widetilde{Z}_{s}\right) d W_{s}\right)\right] \\
& \leq\left.\left. 3 \mathbb{E}\left|\int_{0}^{t_{1} \wedge \sigma_{R}}\right| Q_{s}\right|^{2}\left\|g\left(\bar{r}(s), \hat{Z}_{s}, \widetilde{Z}_{s}\right)\right\|^{2} d s\right|^{1 / 2} \\
& \leq \frac{1}{4} \mathbb{E}\left[\sup _{0 \leq t \leq t_{1}}\left|Q_{t}\right|^{2}\right]+9 \mathbb{E} \int_{0}^{t \wedge \sigma_{R}}\left\|g\left(\bar{r}(s), \hat{Z}_{s}, \widetilde{Z}_{s}\right)\right\|_{2}^{2} d t \\
& \leq \frac{1}{4} \mathbb{E}\left[\sup _{0 \leq t \leq t_{1}}\left|Q_{t}\right|^{2}\right]+18 L^{2} \int_{0}^{t \wedge \sigma_{R}} \mathbb{E}\left(\left\|\hat{Z}_{s}\right\|_{C}^{2}+\left\|\widetilde{Z}_{s}\right\|_{C}^{2}\right) d t .
\end{aligned}
$$

Substituting this into 4.19 yields

$$
\begin{aligned}
\mathbb{E}\left[\sup _{-\tau \leq t \leq t_{1}}\left|Q_{t \wedge \sigma_{R}}\right|^{2}\right] \leq & \mathbb{E}\left[\sup _{-\tau \leq s \leq 0}|\varphi(s, a)|^{2}\right]+\left(K_{5}+36 L^{2}\left(C_{2}+C_{3}\right)\right) \int_{0}^{t_{1} \wedge \sigma_{R}} \mathbb{E}\left[\sup _{-\tau \leq u \leq s}\left|Q_{u}\right|^{2} d s\right] \\
& +\frac{1}{2} \mathbb{E}\left[\sup _{-\tau \leq t \leq t_{1}}\left|Q_{t \wedge \sigma_{R}}\right|^{2}\right]+\left(36 L^{2}+K_{2}\right) C_{4} T
\end{aligned}
$$

Applying the Gronwall inequality, we get

$$
\mathbb{E}\left[\sup _{-\tau \leq t \leq t_{1}}\left|Q_{t \wedge \sigma_{R}}\right|^{2}\right] \leq C_{5}, \quad t_{1} \in[0, T] .
$$

Thus, the proof is complete.

Next, we shall employ the technique in [27] to bound the errors of replacing the right85 continuous Markov chain by the interpolation of the discrete time Markov chain.

Lemma 4.6. Let $\Delta<\min \left\{1,1 /\left(\sqrt{12\left(\bar{\alpha}^{2}+2 L^{2}\right)}\right)\right\}$, for any $t \in[0, T]$

$$
\begin{aligned}
& \mathbb{E} \int_{0}^{t \wedge v_{R}}\left|f\left(\bar{r}(s), \bar{Z}_{s}, \widetilde{Z}_{s}\right)-f\left(r(s), \bar{Z}_{s}, \widetilde{Z}_{s}\right)\right|^{2} d s \leq C_{6} \Delta, \\
& \mathbb{E} \int_{0}^{t \wedge v_{R}}\left|f\left(\bar{r}(s), \hat{Z}_{s}, \widetilde{Z}_{s}\right)-f\left(r(s), \hat{Z}_{s}, \widetilde{Z}_{s}\right)\right|^{2} d s \leq C_{7} \Delta, \\
& \mathbb{E} \int_{0}^{t \wedge v_{R}}\left\|g\left(\bar{r}(s), \hat{Z}_{s}, \widetilde{Z}_{s}\right)-g\left(r(s), \hat{Z}_{s}, \widetilde{Z}_{s}\right)\right\|_{2}^{2} d s \leq C_{8} \Delta,
\end{aligned}
$$

where $\tau_{R}=\inf \left\{t \geq 0:\left|P_{t}\right| \geq R\right\}, \sigma_{R}=\inf \left\{t \geq 0:\left|Q_{t}\right| \geq R\right\}, v_{R}=\tau_{R} \wedge \sigma_{R}$ and $C_{6}, C_{7}, C_{8}$ is independent of $\Delta$. 


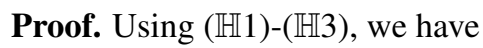

$$
\mathbb{E} \int_{0}^{t \wedge v_{R}}\left|f\left(\bar{r}(s), \bar{Z}_{s}, \widetilde{Z}_{s}\right)-f\left(r(s), \bar{Z}_{s}, \widetilde{Z}_{s}\right)\right|^{2} d s \leq \sum_{k=0}^{\lfloor t / \Delta\rfloor} M_{k}
$$

where

$$
\begin{aligned}
M_{k} & :=\mathbb{E} \int_{t_{k} \wedge v_{R}}^{t_{k+1} \wedge v_{R}}\left|f\left(\bar{r}(s), Q_{k}, Q_{\lfloor\delta(k \Delta) / \Delta\rfloor}\right)-f\left(r(s), Q_{k}, Q_{\lfloor\delta(k \Delta) / \Delta\rfloor}\right)\right|^{2} d s \\
& =\mathbb{E} \int_{t_{k} \wedge v_{R}}^{t_{k+1} \wedge v_{R}}\left|f\left(\bar{r}(s), Q_{k}, Q_{\lfloor\delta(k \Delta) / \Delta\rfloor}\right)-f\left(r(s), Q_{k}, Q_{\lfloor\delta(k \Delta) / \Delta\rfloor}\right)\right|^{2} \mathbb{I}_{\left\{r(s) \neq r\left(t_{k}\right)\right\}}(s) d s \\
& \leq 4 L^{2} \mathbb{E} \int_{t_{k} \wedge v_{R}}^{t_{k+1} \wedge v_{R}}\left[\left|Q_{k}\right|^{2}+\left|Q_{\lfloor\delta(k \Delta) / \Delta\rfloor}\right|^{2}\right] \mathbb{I}_{\left\{r(s) \neq r\left(t_{k}\right)\right\}}(s) d s \\
& =4 L^{2} \int_{t_{k} \wedge v_{R}}^{t_{k+1} \wedge v_{R}} \mathbb{E}\left[\mathbb{E}\left[\left|Q_{k}\right|^{2}+\left|Q_{\lfloor\delta(k \Delta) / \Delta\rfloor}\right|^{2}\right] \mid r\left(t_{k}\right)\right] \mathbb{E}\left[\mathbb{I}_{\left\{r(s) \neq r\left(t_{k}\right)\right\}}(s) \mid r\left(t_{k}\right)\right] d s .
\end{aligned}
$$

In the last step we use the fact that $Q_{k}, Q_{\lfloor\delta(k \Delta) / \Delta\rfloor}$ and $r(s) \neq r\left(t_{k}\right)$ are conditionally independent with respect to the $\sigma$-algebra generated by $r\left(t_{k}\right)$. But, by the Markov property,

$$
\begin{aligned}
\mathbb{E}\left[\mathbb{I}_{\left\{r(s) \neq r\left(t_{k}\right)\right\}}(s) \mid r\left(t_{k}\right)\right] & =\sum_{i \in S} P\left(r\left(t_{k}\right)=i\right) P\left(r(s) \neq r\left(t_{k}\right) \mid r\left(t_{k}\right)=i\right) \\
& =\sum_{i \in S} P\left(r\left(t_{k}\right)=i\right) \sum_{j \neq i} P\left(r(s)=j \mid r\left(t_{k}\right)=i\right) \\
& \leq \sum_{i \in S} P\left(r\left(t_{k}\right)=i\right) \sum_{j \neq i}\left(\gamma_{i j} \Delta+o(\Delta)\right) \\
& =\sum_{i \in S} P\left(r\left(t_{k}\right)=i\right)\left(\left(-\gamma_{i i}\right) \Delta+o(\Delta)\right) \\
& \leq \hat{\gamma} \Delta,
\end{aligned}
$$

where $\hat{\gamma}=\left[N \max _{1 \leq i \leq N}\left(-\gamma_{i i}\right)\right]$. Substituting this into 4.23 along with Lemma 4.5 gives

$$
M_{k} \leq 4 L^{2} \Delta \int_{t_{k} \wedge v_{R}}^{t_{k+1} \wedge v_{R}} \mathbb{E}\left[\left|Q_{k}\right|^{2}+\left|Q_{[\delta(k \Delta) / \Delta\rfloor}\right|^{2}\right] d s \leq 8 L^{2} C_{5} \hat{\gamma} \Delta^{2} .
$$

Combining (4.22) and 4.24, we obtain that

$$
\mathbb{E} \int_{0}^{t \wedge v_{R}}\left|f\left(\bar{r}(s), \bar{Z}_{s}, \widetilde{Z}_{s}\right)-f\left(r(s), \bar{Z}_{s}, \widetilde{Z}_{s}\right)\right|^{2} d s \leq 8 L^{2} C_{5} \hat{\gamma} T \Delta=C_{6} \Delta .
$$

Similarly, we can prove that

$$
\begin{aligned}
& \mathbb{E} \int_{0}^{t \wedge v_{R}}\left|f\left(\bar{r}(s), \bar{Z}_{s}, \widetilde{Z}_{s}\right)-f\left(r(s), \hat{Z}_{s}, \widetilde{Z}_{s}\right)\right|^{2} d s \leq C_{7} \Delta, \\
& \mathbb{E} \int_{0}^{t \wedge v_{R}}\left\|g\left(\bar{r}(s), \bar{Z}_{s}, \widetilde{Z}_{s}\right)-g\left(r(s), \hat{Z}_{s}, \widetilde{Z}_{s}\right)\right\|_{2}^{2} d s \leq C_{8} \Delta .
\end{aligned}
$$

Thus, the proof is complete. 
Lemma 4.7. Under the assumptions $(\mathbb{H} 1)-(\mathbb{H} 3)$ and let $\Delta<\min \left\{1,1 /\left(\sqrt{12\left(\bar{\alpha}^{2}+2 L^{2}\right)}\right)\right\}$, for any $k \geq 2$, there is a positive constant $C_{9}$ such that

$$
\mathbb{E}\left[\sup _{-\tau \leq t \leq T}\left|Q_{t \wedge \sigma_{R}}\right|^{k}\right] \leq C_{9}
$$

where $\sigma_{R}=\inf \left\{t \geq 0:\left|Q_{t}\right| \geq R\right\}$ and $C_{9}$ is independent of $\Delta$.

so The proof is similar to that of Lemma 4.3.

Lemma 4.8. Under the Assumptions $(\mathbb{H} 1)-(\mathbb{H} 3)$ and let $\Delta<\min \left\{1,1 /\left(\sqrt{12\left(\bar{\alpha}^{2}+2 L^{2}\right)}\right)\right\}, \mathbb{E}\left|\frac{\partial Q_{n}}{\partial a}\right|^{2}<$ $\infty$, there exist positive constants $C_{10}$ and $C_{11}$ independent of $\Delta$ such that

$$
\begin{aligned}
& \mathbb{E}\left[\sup _{0 \leq t \leq T}\left|Q_{t}-\bar{Z}_{t}\right|^{2}\right] \leq C_{10} \Delta, \\
& \mathbb{E}\left[\sup _{0 \leq t \leq T}\left|Q_{t}-\hat{Z}_{t}\right|^{2}\right] \leq C_{11} \Delta .
\end{aligned}
$$

Proof. For $t \in[0, T]$, there exists an integer $k$ such that $t \in[k \Delta,(k+1) \Delta)$, we have

$$
\begin{aligned}
Q_{t}-\bar{Z}_{t}= & -\int_{k \Delta}^{t} \frac{\partial Q_{k}}{\partial a} d s+\int_{k \Delta}^{t}\left((1-\theta) f\left(\bar{r}(s), \bar{Z}_{s}, \widetilde{Z}_{s}\right)+\theta f\left(\bar{r}(s), \hat{Z}_{s}, \widetilde{Z}_{s}\right)\right) d s \\
& -\int_{k \Delta}^{t} \mu(s, a)\left((1-\theta) \bar{Z}_{s}+\theta \hat{Z}_{s}\right) d s+\int_{k \Delta}^{t} g\left(\bar{r}(s), \hat{Z}_{s}, \widetilde{Z}_{s}\right) d W_{s} .
\end{aligned}
$$

Applying $(a+b+c+d)^{2} \leq 4 a^{2}+4 b^{2}+4 c^{2}+4 d^{2}$ and conditions (H11)-( $\left.\mathbb{H} 2\right)$, we find that

$$
\begin{aligned}
\left|Q_{t}-\bar{Z}_{t}\right|^{2} \leq & 4\left|\int_{k \Delta}^{t} \frac{\partial Q_{k}}{\partial a} d s\right|^{2}+4\left|\int_{k \Delta}^{t}\left((1-\theta) f\left(\bar{r}(s), \bar{Z}_{s}, \widetilde{Z}_{s}\right)+\theta f\left(\bar{r}(s), \hat{Z}_{s}, \widetilde{Z}_{s}\right)\right) d s\right|^{2} \\
& +4\left|\int_{k \Delta}^{t}\left(\mu(s, a)\left((1-\theta) \bar{Z}_{s}+\theta \hat{Z}_{s}\right)\right) d s\right|^{2}+4\left|\int_{k \Delta}^{t} g\left(\bar{r}(s), \hat{Z}_{s}, \widetilde{Z}_{s}\right) d W_{s}\right|^{2} \\
\leq & 4 \Delta \int_{k \Delta}^{t}\left|\frac{\partial Q_{k}}{\partial a}\right|^{2} d s+4 \Delta \int_{k \Delta}^{t}\left|\left((1-\theta) f\left(\bar{r}(s), \bar{Z}_{s}, \widetilde{Z}_{s}\right)+\theta f\left(\bar{r}(s), \hat{Z}_{s}, \widetilde{Z}_{s}\right)\right)\right|^{2} d s \\
& +4 \bar{\alpha}^{2} \Delta \int_{k \Delta}^{t}\left|(1-\theta) \bar{Z}_{s}+\theta \hat{Z}_{s}\right|^{2} d s+4\left|\int_{k \Delta}^{t} g\left(\bar{r}(s), \hat{Z}_{s}, \widetilde{Z}_{s}\right) d W_{s}\right|^{2} \\
\leq & 4 \Delta \int_{k \Delta}^{t}\left|\frac{\partial Q_{k}}{\partial a}\right|^{2} d s+8 L^{2} \Delta \int_{k \Delta}^{t}\left(\left\|\bar{Z}_{s}\right\|_{C}^{2}+|| \widetilde{Z}_{s}\left\|_{C}^{2}+\right\| \hat{Z}_{C}^{2}+\left\|\widetilde{Z}_{s}\right\|_{C}^{2}\right) d s \\
& +4 \bar{\alpha}^{2} \Delta \int_{k \Delta}^{t}\left(\left|\bar{Z}_{s}\right|^{2}+\left|\hat{Z}_{s}\right|^{2}\right) d s+4\left|\int_{k \Delta}^{t} g\left(\bar{r}(s), \hat{Z}_{s}, \widetilde{Z}_{s}\right) d W_{s}\right|^{2} .
\end{aligned}
$$

Taking expectations, we have

$$
\begin{aligned}
\mathbb{E}\left[\sup _{0 \leq t \leq T}\left|Q_{t}-\bar{Z}_{t}\right|^{2}\right] \leq & \mathbb{E}\left[\max _{0 \leq k \leq M-1} \sup _{k \Delta \leq u \leq(k+1) \Delta}\left|Q_{u}-\bar{Z}_{u}\right|^{2}\right] \\
\leq & \max _{0 \leq k \leq M-1}\left(4 \Delta^{2} \mathbb{E}\left|\frac{\partial Q_{k}}{\partial a}\right|^{2}+4 \bar{\alpha}^{2} \Delta \int_{k \Delta}^{(k+1) \Delta}\left(\mathbb{E}\left|\bar{Z}_{s}\right|^{2}+\mathbb{E}\left|\hat{Z}_{s}\right|^{2}\right) d s\right. \\
& \left.+8 L^{2} \Delta \int_{k \Delta}^{(k+1) \Delta}\left(\mathbb{E}|| \bar{Z}_{s}\left\|_{C}^{2}+\mathbb{E}|| \widetilde{Z}_{s}\right\|_{C}^{2}+\mathbb{E}|| \hat{Z}\left\|_{C}^{2}+\mathbb{E}|| \widetilde{Z}_{s}\right\|_{C}^{2}\right) d s\right) \\
& +4 \mathbb{E}\left[\max _{0 \leq k \leq M-1} \sup _{k \Delta \leq u \leq(k+1) \Delta}\left|\int_{k \Delta}^{u} g\left(\bar{r}(s), \hat{Z}_{s}, \widetilde{Z}_{s}\right) d W_{s}\right|^{2}\right] .
\end{aligned}
$$


Using the Doob martingale inequality (see e.g. [6, 11]), Lemma 4.4 and 4.5, we have

$$
\begin{aligned}
\mathbb{E}\left[\sup _{0 \leq t \leq T}\left|Q_{t}-\bar{Z}_{t}\right|^{2}\right] & \leq 16 \max _{0 \leq k \leq M-1}\left[\int_{k \Delta}^{(k+1) \Delta} \mathbb{E}\left\|g\left(\bar{r}(s), \hat{Z}_{s}, \widetilde{Z}_{s}\right)\right\|_{2}^{2} d s\right]+o(\Delta) \\
& \leq 32 L^{2} \max _{0 \leq k \leq M-1}\left[\int_{k \Delta}^{(k+1) \Delta}\left(\mathbb{E}|| \hat{Z}_{s}\left\|_{C}^{2}+\mathbb{E}|| \widetilde{Z}_{s}\right\|_{C}^{2}\right) d s\right]+o(\Delta) \\
& \leq 32 L^{2} \Delta \max _{0 \leq k \leq M-1}\left(C_{2} \mathbb{E}\left|Q_{k}\right|^{2}+\left(C_{3}+1\right) \mathbb{E}\left|Q_{\lfloor\delta(k \Delta) / \Delta\rfloor}\right|^{2}+C_{4}\right)+o(\Delta) \\
& \leq 32 L^{2}\left(\left(C_{2}+C_{3}+1\right) C_{9}+C_{4}\right) \Delta+o(\Delta) .
\end{aligned}
$$

A similar analysis gives

$$
\mathbb{E}\left[\sup _{0 \leq t \leq T}\left|Q_{t}-\hat{Z}_{t}\right|^{2}\right] \leq C_{11} \Delta
$$

Thus, the proof is complete.

Next, we will employ the method due to Mao [16] to prove the following lemma.

Lemma 4.9. Under the assumptions $(\mathbb{H} 1)-(\mathbb{H} 3)$ and let $\Delta<\min \left\{1,1 /\left(\sqrt{12\left(\bar{\alpha}^{2}+2 L^{2}\right)}\right)\right\}, \mathbb{E}\left|\frac{\partial Q_{n}}{\partial a}\right|^{2}<$ $\infty$, there exists a positive constant $C_{12}$ independent of $\Delta$ such that

$$
\mathbb{E}\left[\sup _{0 \leq t \leq T}\left|Q_{\delta(t)}-\widetilde{Z}_{t}\right|^{2}\right] \leq C_{12} \Delta .
$$

Proof. For $t \in[0, T]$, there exists an integer $k$ such that $t \in[k \Delta,(k+1) \Delta)$. By $(2.2)$ and the triangle inequality, we yield

$$
\begin{aligned}
|\delta(t)-\lfloor\delta(k \Delta) / \Delta\rfloor \Delta| & \leq|\delta(t)-\delta(k \Delta)|+|\delta(k \Delta)-\lfloor\delta(k \Delta) / \Delta\rfloor \Delta| \\
& \leq \rho \Delta+|\delta(k \Delta) / \Delta-\lfloor\delta(k \Delta) / \Delta\rfloor| \Delta \\
& \leq(\rho+1) \Delta .
\end{aligned}
$$

To show the desired result, let us consider the following four possible cases:

- If $\delta(t) \geq\lfloor\delta(k \Delta) / \Delta\rfloor \Delta \geq 0$ or $\lfloor\delta(k \Delta) / \Delta\rfloor \Delta \geq \delta(t) \geq 0$, we have

$$
\begin{aligned}
\left|Q_{\delta(t)}-\widetilde{Z}_{t}\right| & \\
= & -\int_{\lfloor\delta(k \Delta) / \Delta\rfloor \Delta}^{\delta(t)} \frac{\partial Q_{k}}{\partial a} d s+\int_{\lfloor\delta(k \Delta) / \Delta\rfloor \Delta}^{\delta(t)}\left((1-\theta) f\left(\bar{r}(s), \bar{Z}_{s}, \widetilde{Z}_{s}\right)+\theta f\left(\bar{r}(s), \hat{Z}_{s}, \widetilde{Z}_{s}\right)\right) d s \\
& -\int_{\lfloor\delta(k \Delta) / \Delta\rfloor \Delta}^{\delta(t)} \mu(s, a)\left((1-\theta) \bar{Z}_{s}+\theta \hat{Z}_{s}\right) d s+\int_{\lfloor\delta(k \Delta) / \Delta\lrcorner \Delta}^{\delta(t)} g\left(\bar{r}(s), \hat{Z}_{s}, \widetilde{Z}_{s}\right) d W_{s} \mid .
\end{aligned}
$$

Applying $(a+b+c+d)^{2} \leq 4 a^{2}+4 b^{2}+4 c^{2}+4 d^{2}$ and (4.26), we have

$$
\begin{aligned}
& \left|Q_{\delta(t)}-\widetilde{Z}_{t}\right|^{2} \\
& \leq 4\left|\int_{[\delta(k \Delta) / \Delta\rfloor \Delta}^{\delta(t)} \frac{\partial Q_{k}}{\partial a} d s\right|^{2}+4\left|\int_{[\delta(k \Delta) / \Delta\rfloor \Delta}^{\delta(t)}\left((1-\theta) f\left(\bar{r}(s), \bar{Z}_{s}, \widetilde{Z}_{s}\right)+\theta f\left(\bar{r}(s), \hat{Z}_{s}, \widetilde{Z}_{s}\right)\right) d s\right|^{2} \\
& \quad+4\left|\int_{\lfloor\delta(k \Delta) / \Delta\rfloor \Delta}^{\delta(t)}\left(\mu(s, a)\left((1-\theta) \bar{Z}_{s}+\theta \hat{Z}_{s}\right)\right) d s\right|^{2}+4\left|\int_{[\delta(k \Delta) / \Delta\rfloor \Delta}^{\delta(t)} g\left(\bar{r}(s), \hat{Z}_{s}, \widetilde{Z}_{s}\right) d W_{s}\right|^{2}
\end{aligned}
$$




$$
\begin{aligned}
\leq & 4(\rho+1) \Delta \int_{[\delta(k \Delta) / \Delta\rfloor \Delta}^{\delta(t)}\left|\frac{\partial Q_{k}}{\partial a}\right|^{2} d s+8 L^{2}(\rho+1) \Delta \int_{[\delta(k \Delta) / \Delta\rfloor \Delta}^{\delta(t)}\left(\left\|\bar{Z}_{s}\right\|_{C}^{2}+2 \mid\left\|\widetilde{Z}_{s}\right\|_{C}^{2}+\|\hat{Z}\|_{C}^{2}\right) d s \\
& +4 \bar{\alpha}^{2}(\rho+1) \Delta \int_{[\delta(k \Delta) / \Delta \Delta \Delta}^{\delta(t)}\left(\left|\bar{Z}_{s}\right|^{2}+\left|\hat{Z}_{s}\right|^{2}\right) d s+4\left|\int_{[\delta(k \Delta) / \Delta\rfloor \Delta}^{\delta(t)} g\left(\bar{r}(s), \hat{Z}_{s}, \widetilde{Z}_{s}\right) d W_{s}\right|^{2} .
\end{aligned}
$$

Taking expectations and using Lemma 4.4 and 4.5 as well as 4.26, we have

$$
\begin{aligned}
\mathbb{E} & {\left[\sup _{0 \leq t \leq T}\left|Q_{\delta(t)}-\widetilde{Z}_{t}\right|^{2}\right] } \\
\leq & \mathbb{E}\left[\max _{0 \leq k \leq M-1} \sup _{k \Delta \leq u \leq(k+1) \Delta}\left|Q_{\delta(u)}-\widetilde{Z}_{u}\right|^{2}\right] \\
\leq & \max _{0 \leq k \leq M-1}\left(4(\rho+1)^{2} \Delta^{2} \mathbb{E}\left|\frac{\partial Q_{k}}{\partial a}\right|^{2}+4 \bar{\alpha}^{2}(\rho+1) \Delta \int_{[\delta(k \Delta) / \Delta\rfloor \Delta}^{\lfloor\delta(k \Delta) / \Delta\rfloor \Delta+(\rho+1) \Delta}\left(\mathbb{E}\left|\bar{Z}_{s}\right|^{2}+\mathbb{E}\left|\hat{Z}_{s}\right|^{2}\right) d s\right. \\
& \left.+8 L^{2}(\rho+1) \Delta \int_{[\delta(k \Delta) / \Delta\rfloor \Delta}^{\lfloor\delta(k \Delta) / \Delta\lrcorner \Delta+(\rho+1) \Delta}\left(\mathbb{E}|| \bar{Z}_{s}\left\|_{C}^{2}+2 \mathbb{E}|| \widetilde{Z}_{s}\right\|_{C}^{2}+\mathbb{E}\|\mid \hat{Z}\|_{C}^{2}\right) d s\right) \\
& +4 \mathbb{E}\left[\max _{0 \leq k \leq M-1} \sup _{k \Delta \leq u \leq(k+1) \Delta}\left|\int_{[\delta(k \Delta) / \Delta \Delta \Delta}^{\delta(u)} g\left(\bar{r}(s), \hat{Z}_{s}, \widetilde{Z}_{s}\right) d W_{s}\right|^{2}\right] \\
\leq & 4 \mathbb{E}\left[\max _{0 \leq k \leq M-1} \sup _{k \Delta \leq u \leq(k+1) \Delta}\left|\int_{[\delta(k \Delta) / \Delta\lrcorner \Delta}^{\delta(u)} g\left(\bar{r}(s), \hat{Z}_{s}, \widetilde{Z}_{s}\right) d W_{s}\right|^{2}\right]+o(\Delta) .
\end{aligned}
$$

Using the Doob martingale inequality along with Lemma 4.4 and 4.5, we have

$$
\begin{aligned}
& \mathbb{E}\left[\sup _{0 \leq t \leq T}\left|Q_{\delta(t)}-\widetilde{Z}_{t}\right|^{2}\right] \\
& \leq 16 \max _{0 \leq k \leq M-1}\left[\int_{[\delta(k \Delta) / \Delta\rfloor \Delta}^{\lfloor\delta(k \Delta) / \Delta\rfloor \Delta+(\rho+1) \Delta} \mathbb{E}\left\|g\left(\bar{r}(s), \hat{Z}_{s}, \widetilde{Z}_{s}\right)\right\|_{2}^{2} d s\right]+o(\Delta) \\
& \leq 32 L^{2} \max _{0 \leq k \leq M-1}\left[\int_{[\delta(k \Delta) / \Delta\rfloor \Delta}^{\lfloor\delta(k \Delta) / \Delta\rfloor \Delta+(\rho+1) \Delta}\left(\mathbb{E}|| \hat{Z}_{s}\left\|_{C}^{2}+\mathbb{E} \mid\right\| \widetilde{Z}_{s} \|_{C}^{2}\right) d s\right]+o(\Delta) \\
& \leq 32 L^{2}(\rho+1) \Delta \max _{0 \leq k \leq M-1}\left(C_{2} \mathbb{E}\left|Q_{k}\right|^{2}+\left(C_{3}+1\right) \mathbb{E}\left|Q_{[\delta(k \Delta) / \Delta\rfloor}\right|^{2}+C_{4}\right)+o(\Delta) \\
& \leq 32 L^{2}(\rho+1)\left(\left(C_{2}+C_{3}+1\right) C_{9}+C_{4}\right) \Delta+o(\Delta) .
\end{aligned}
$$

- If $\delta(t) \leq\lfloor\delta(k \Delta) / \Delta\rfloor \Delta \leq 0$ or $\lfloor\delta(k \Delta) / \Delta\rfloor \Delta \leq \delta(t) \leq 0$. Then, by 3.1) and 3.2), we have

$$
Q_{\delta(t)}-\widetilde{Z}_{t}=\varphi(\delta(t), a)-\varphi(\lfloor\delta(k \Delta) / \Delta\rfloor \Delta, a) .
$$

By 2.2, 2.3 and (4.26, we get

$$
\begin{aligned}
& \mathbb{E}\left[\sup _{0 \leq t \leq T}\left|Q_{\delta(t)}-\widetilde{Z}_{t}\right|^{2}\right] \\
& \leq \mathbb{E}\left[\max _{0 \leq k \leq M-1} \sup _{k \Delta \leq u \leq(k+1) \Delta}\left|Q_{\delta(u)}-\widetilde{Z}_{u}\right|^{2}\right] \\
& \leq \mathbb{E}\left[\max _{0 \leq k \leq M-1} \sup _{-\tau \leq \delta(u), L \delta(k \Delta) / \Delta\rfloor \Delta \leq 0}|\varphi(\delta(t), a)-\varphi(\lfloor\delta(k \Delta) / \Delta\rfloor \Delta, a)|^{2}\right] \\
& \leq \bar{K}|\delta(t)-\lfloor\delta(k \Delta) / \Delta\rfloor \Delta| \\
& \leq \bar{K}(\rho+1) \Delta .
\end{aligned}
$$


- If $\lfloor\delta(k \Delta) / \Delta\rfloor \Delta \leq 0 \leq \delta(t)$. Then, $-\lfloor\delta(k \Delta) / \Delta\rfloor \Delta \leq(\rho+1) \Delta, \delta(t) \leq(\rho+1) \Delta$. Hence

$$
\begin{aligned}
& \mathbb{E}\left[\sup _{0 \leq t \leq T}\left|Q_{\delta(t)}-\widetilde{Z}_{t}\right|^{2}\right] \\
& \leq 2 \mathbb{E}\left[\sup _{0 \leq t \leq T}\left|Q_{\delta(t)}-Q_{0}\right|^{2}\right]+2 \mathbb{E}\left[\sup _{0 \leq t \leq T}\left|\widetilde{Z}_{t}-\varphi(0, a)\right|^{2}\right] \\
& \left.\leq 2 \mathbb{E}\left[\sup _{0 \leq t \leq T}\left|Q_{\delta(t)}-Q_{0}\right|^{2}\right]+\left.2 \mathbb{E}\left[\max _{0 \leq k \leq M-1} \sup _{-\tau \leq L(k \Delta) / \Delta\lrcorner \Delta \leq 0} \mid \varphi(0, a)-\varphi(L \delta(k \Delta) / \Delta\rfloor \Delta, a\right)\right|^{2}\right] \\
& \leq 64 L^{2}(\rho+1)\left(\left(C_{2}+C_{3}+1\right) C_{9}+C_{4}\right) \Delta+2 \bar{K}(\rho+1) \Delta+o(\Delta) .
\end{aligned}
$$

- If $\delta(t) \leq 0 \leq\lfloor\delta(k \Delta) / \Delta\rfloor \Delta$. Then, we have

$$
\begin{aligned}
& \mathbb{E}\left[\sup _{0 \leq t \leq T}\left|Q_{\delta(t)}-\widetilde{Z}_{t}\right|^{2}\right] \\
& \leq 2 \mathbb{E}\left[\sup _{0 \leq t \leq T}\left|Q_{\delta(t)}-Q_{0}\right|^{2}\right]+2 \mathbb{E}\left[\sup _{0 \leq t \leq T}\left|\widetilde{Z}_{t}-\varphi(0, a)\right|^{2}\right] \\
& \leq 2 \mathbb{E}\left[\sup _{-\tau \leq \delta(t) \leq 0}|\varphi(\delta(0), a)-\varphi(\delta(t), a)|^{2}\right]+2 \mathbb{E}\left[\sup _{0 \leq t \leq T}\left|\widetilde{Z}_{t}-Q_{0}\right|^{2}\right] \\
& \leq 2 \bar{K}(\rho+1) \Delta+64 L^{2}(\rho+1)\left(\left(C_{2}+C_{3}+1\right) C_{9}+C_{4}\right) \Delta+o(\Delta) .
\end{aligned}
$$

Combining these different cases together, we get

$$
\mathbb{E}\left[\sup _{0 \leq t \leq T}\left|Q_{\delta(t)}-\widetilde{Z}_{t}\right|^{2}\right] \leq C_{12} \Delta,
$$

as required.

Theorem 4.10. Under the assumptions $(\mathbb{H} 1)-(\mathbb{H} 3)$ and let $\Delta<\min \left\{1,1 /\left(\sqrt{12\left(\bar{\alpha}^{2}+2 L^{2}\right)}\right)\right\}$, then

$$
\mathbb{E}\left[\sup _{0 \leq t \leq T}\left|P_{t \wedge v_{R}}-Q_{t \wedge v_{R}}\right|^{2}\right] \leq C_{13} \Delta,
$$

where $\tau_{R}=\inf \left\{t \geq 0:\left|P_{t}\right| \geq R\right\}, \sigma_{R}=\inf \left\{t \geq 0:\left|Q_{t}\right| \geq R\right\}, v_{R}=\tau_{R} \wedge \sigma_{R}$ and $C_{13}$ is independent of $\Delta$.

Proof. By (2.1) and 3.3 , we have

$$
\begin{aligned}
P_{t \wedge v_{R}}-Q_{t \wedge \nu_{R}}= & -\int_{0}^{t \wedge v_{R}} \frac{\partial\left(P_{s}-Q_{s}\right)}{\partial a} d s-\int_{0}^{t \wedge v_{R}} \mu(s, a)\left[(1-\theta)\left(P_{s}-\bar{Z}_{s}\right)+\theta\left(P_{s}-\hat{Z}_{s}\right] d s\right. \\
& +\int_{0}^{t \wedge \nu_{R}}\left[(1-\theta)\left(f\left(r(s), P_{s}, P_{\delta(s)}\right)-f\left(\bar{r}(s), \bar{Z}_{s}, \widetilde{Z}_{s}\right)\right)\right] d s \\
& +\int_{0}^{t \wedge v_{R}} \theta\left(f\left(r(s), P_{s}, P_{\delta(s)}\right)-f\left(\bar{r}(s), \hat{Z}_{s}, \widetilde{Z}_{s}\right) d s\right. \\
& +\int_{0}^{t \wedge v_{R}}\left(g\left(r(s), P_{s}, P_{\delta(s)}\right)-g\left(\bar{r}(s), \hat{Z}_{s}, \widetilde{Z}_{s}\right)\right) d W_{s}
\end{aligned}
$$


Using the generalized Itô formula yields

$$
\begin{aligned}
\left|P_{t \wedge v_{R}}-Q_{t \wedge v_{R}}\right|^{2}= & -2 \int_{0}^{t \wedge v_{R}}\left\langle P_{s}-Q_{s}, \frac{\partial\left(P_{s}-Q_{s}\right)}{\partial a}\right\rangle d s \\
& -2 \int_{0}^{t \wedge v_{R}}\left(P_{s}-Q_{s}, \mu(s, a)\left[(1-\theta)\left(P_{s}-\bar{Z}_{s}\right)+\theta\left(P_{s}-\hat{Z}_{s}\right)\right] d s\right) \\
& +2 \int_{0}^{t \wedge v_{R}}\left(P_{s}-Q_{s},(1-\theta)\left(f\left(r(s), P_{s}, P_{\delta(s)}\right)-f\left(\bar{r}(s), \bar{Z}_{s}, \widetilde{Z}_{s}\right)\right)\right) d s \\
& +2 \int_{0}^{t \wedge v_{R}}\left(P_{s}-Q_{s}, \theta\left(f\left(r(s), P_{s}, P_{\delta(s)}\right)-f\left(\bar{r}(s), \hat{Z}_{s}, \widetilde{Z}_{s}\right)\right)\right) d s \\
& +\int_{0}^{t \wedge \nu_{R}}\left\|g\left(r(s), P_{s}, P_{\delta(s)}\right)-g\left(\bar{r}(s), \hat{Z}_{s}, \widetilde{Z}_{s}\right)\right\|_{2}^{2} d s \\
& +2 \int_{0}^{t \wedge v_{R}}\left(P_{s}-Q_{s},\left(g\left(r(s), P_{s}, P_{\delta(s)}\right)-g\left(\bar{r}(s), \hat{Z}_{s}, \widetilde{Z}_{s}\right)\right) d W_{s}\right) .
\end{aligned}
$$

Let

$$
\begin{aligned}
J_{4}(s) & :=f\left(r(s), \bar{Z}_{s}, \bar{Z}_{\delta(s)}\right)-f\left(\bar{r}(s), \bar{Z}_{s}, \widetilde{Z}_{s}\right), \\
J_{5}(s) & :=f\left(r(s), \hat{Z}_{s}, \bar{Z}_{\delta(s)}\right)-f\left(\bar{r}(s), \hat{Z}_{s}, \widetilde{Z}_{s}\right), \\
J_{6}(s) & :=g\left(r(s), \hat{Z}_{s}, \bar{Z}_{\delta(s)}\right)-g\left(\bar{r}(s), \hat{Z}_{s}, \widetilde{Z}_{s}\right) .
\end{aligned}
$$

Lemma 4.1 gives

$$
2 \int_{0}^{t \wedge v_{R}}\left\langle P_{s}-Q_{s}, \frac{\partial\left(P_{s}-Q_{s}\right)}{\partial a}\right\rangle d s \leq A \bar{\beta}^{2} \int_{0}^{t \wedge v_{R}}\left|P_{s}-Q_{s}\right|^{2} d s .
$$

By $(\mathbb{H} 1)-(\mathbb{H} 3)$ and the elementary inequalities

$$
2\langle u, v\rangle \leq|u|^{2}+|v|^{2}, \quad|(1-\theta) u+\theta v|^{2} \leq|u|^{2}+|v|^{2}, \quad u, v \in \mathbb{R}^{n},
$$

we get

$$
\begin{aligned}
& -2 \int_{0}^{t \wedge v_{R}}\left(P_{s}-Q_{s}, \mu(s, a)\left[(1-\theta)\left(P_{s}-\bar{Z}_{s}\right)+\theta\left(P_{s}-\hat{Z}_{s}\right)\right]\right) d s \\
& \leq \bar{\alpha} \int_{0}^{t \wedge v_{R}}\left(\left|P_{s}-Q_{s}\right|^{2}+\left|P_{s}-\bar{Z}_{s}\right|^{2}+\left|P_{s}-\hat{Z}_{s}\right|^{2}\right) d s \\
& \leq \bar{\alpha} \int_{0}^{t \wedge v_{R}}\left(\left|P_{s}-Q_{s}\right|^{2}+2\left|P_{s}-Q_{s}\right|^{2}+2\left|Q_{s}-\bar{Z}_{s}\right|^{2}+2\left|P_{s}-Q_{s}\right|^{2}+2\left|Q_{s}-\hat{Z}_{s}\right|^{2}\right) d s \\
& \leq 5 \bar{\alpha} \int_{0}^{t \wedge v_{R}}\left|P_{s}-Q_{s}\right|^{2} d s+2 \bar{\alpha} \int_{0}^{t \wedge v_{R}}\left|Q_{s}-\bar{Z}_{s}\right|^{2} d s+2 \bar{\alpha} \int_{0}^{t \wedge v_{R}}\left|Q_{s}-\hat{Z}_{s}\right|^{2} d s .
\end{aligned}
$$

Similarly, we have

$$
\begin{gathered}
2 \int_{0}^{t \wedge v_{R}}\left(P_{s}-Q_{s},(1-\theta)\left(f\left(r(s), P_{s}, P_{\delta(s)}\right)-f\left(\bar{r}(s), \bar{Z}_{s}, \widetilde{Z}_{s}\right)\right)\right) d s \\
\leq \int_{0}^{t \wedge v_{R}}\left(\left|P_{s}-Q_{s}\right|^{2}+2\left|f\left(r(s), P_{s}, P_{\delta(s)}\right)-f\left(r(s), \bar{Z}_{s}, \widetilde{Z}_{s}\right)\right|^{2}\right. \\
17
\end{gathered}
$$




$$
\begin{aligned}
& \left.+2\left|f\left(r(s), \bar{Z}_{s}, \widetilde{Z}_{s}\right)-f\left(\bar{r}(s), \bar{Z}_{s}, \widetilde{Z}_{s}\right)\right|^{2}\right) d s \\
\leq & \int_{0}^{t \wedge v_{R}}\left(\left|P_{s}-Q_{s}\right|^{2}+4 L^{2}\left\|P_{s}-\bar{Z}_{s}\right\|_{C}^{2}+4 L^{2}\left\|P_{\delta(s)}-\widetilde{Z}_{s}\right\|_{C}^{2}+2\left|J_{4}(s)\right|^{2}\right) d s \\
\leq & \int_{0}^{t \wedge \nu_{R}}\left(\left|P_{s}-Q_{s}\right|^{2}+8 L^{2}\left\|P_{s}-Q_{s}\right\|_{C}^{2}+8 L^{2}\left\|Q_{s}-\bar{Z}_{s}\right\|_{C}^{2}\right. \\
& \left.+8 L^{2}\left\|P_{\delta(s)}-Q_{\delta(s)}\right\|_{C}^{2}+8 L^{2}\left\|Q_{\delta(s)}-\widetilde{Z}_{s}\right\|_{C}^{2}+2\left|J_{4}(s)\right|^{2}\right) d s, \\
2 & \int_{0}^{t \wedge v_{R}}\left(P_{s}-Q_{s}, \theta\left(f\left(r(s), P_{s}, P_{\delta(s)}\right)-f\left(\bar{r}(s), \hat{Z}_{s}, \widetilde{Z}_{s}\right)\right)\right) d s \\
\leq & \int_{0}^{t \wedge v_{R}}\left(\left|P_{s}-Q_{s}\right|^{2}+2\left|f\left(r(s), P_{s}, P_{\delta(s)}\right)-f\left(r(s), \hat{Z}_{s}, \widetilde{Z}_{s}\right)\right|^{2}\right. \\
& \left.+2\left|f\left(r(s), \hat{Z}_{s}, \widetilde{Z}_{s}\right)-f\left(\bar{r}(s), \hat{Z}_{s}, \widetilde{Z}_{s}\right)\right|^{2}\right) d s \\
\leq & \int_{0}^{t \wedge v_{R}}\left(\left|P_{s}-Q_{s}\right|^{2}+4 L^{2}\left\|P_{s}-\hat{Z}_{s}\right\|_{C}^{2}+4 L^{2}\left\|P_{\delta(s)}-\widetilde{Z}_{s}\right\|_{C}^{2}+2\left|J_{5}(s)\right|^{2}\right) d s \\
\leq & \int_{0}^{t \wedge v_{R}}\left(\left|P_{s}-Q_{s}\right|^{2}+8 L^{2}\left\|P_{s}-Q_{s}\right\|_{C}^{2}+8 L^{2}\left\|Q_{s}-\hat{Z}_{s}\right\|_{C}^{2}\right. \\
& \left.+8 L^{2}\left\|P_{\delta(s)}-Q_{\delta(s)}\right\|_{C}^{2}+8 L^{2}\left\|Q_{\delta(s)}-\widetilde{Z}_{s}\right\|_{C}^{2}+2\left|J_{5}(s)\right|^{2}\right) d s
\end{aligned}
$$

and

$$
\begin{aligned}
& \int_{0}^{t \wedge v_{R}}\left\|g\left(r(s), P_{s}, P_{\delta(s)}\right)-g\left(\bar{r}(s), \hat{Z}_{s}, \widetilde{Z}_{s}\right)\right\|_{2}^{2} d s \\
& \leq \int_{0}^{t \wedge v_{R}}\left(2\left\|g\left(r(s), P_{s}, P_{\delta(s)}\right)-g\left(r(s), \hat{Z}_{s}, \widetilde{Z}_{s}\right)\right\|_{2}^{2}\right. \\
& \left.\quad+2\left\|g\left(r(s), \hat{Z}_{s}, \widetilde{Z}_{s}\right)-g\left(\bar{r}(s), \hat{Z}_{s}, \widetilde{Z}_{s}\right)\right\|_{C}^{2}\right) d s \\
& \leq \int_{0}^{t \wedge v_{R}}\left(4 L^{2}\left\|P_{s}-\hat{Z}_{s}\right\|_{C}^{2}+4 L^{2}\left\|P_{\delta(s)}-\widetilde{Z}_{s}\right\|_{C}^{2}+2\left|J_{6}(s)\right|^{2}\right) d s \\
& \leq \int_{0}^{t \wedge v_{R}}\left(8 L^{2}\left\|P_{s}-Q_{s}\right\|_{C}^{2}+8 L^{2}\left\|Q_{s}-\hat{Z}_{s}\right\|_{C}^{2}\right. \\
& \left.\quad+8 L^{2}\left\|P_{\delta(s)}-Q_{\delta(s)}\right\|_{C}^{2}+8 L^{2}\left\|Q_{\delta(s)}-\widetilde{Z}_{s}\right\|_{C}^{2}+2\left|J_{6}(s)\right|^{2}\right) d s .
\end{aligned}
$$

95 Substituting (4.28)-4.32) into 4.27), we have

$$
\begin{aligned}
\left|P_{t \wedge v_{R}}-Q_{t \wedge v_{R}}\right|^{2} & \leq \mu_{3} \int_{0}^{t \wedge v_{R}}\left\|P_{s}-Q_{s}\right\|_{C}^{2} d s+\mu_{4} \int_{0}^{t \wedge v_{R}}\left\|Q_{s}-\bar{Z}_{s}\right\|_{C}^{2} d s \\
& +\mu_{5} \int_{0}^{t \wedge v_{R}}\left\|Q_{s}-\hat{Z}_{s}\right\|_{C}^{2} d s+\mu_{6} \int_{0}^{t \wedge v_{R}}\left\|P_{\delta(s)}-Q_{\delta(s)}\right\|_{C}^{2} d s \\
& +\mu_{7} \int_{0}^{t \wedge v_{R}}\left\|Q_{\delta(s)}-\widetilde{Z}_{s}\right\|_{C}^{2} d s+2 \int_{0}^{t \wedge v_{R}}\left(\left.J_{4}(s)\right|^{2}+\left.J_{5}(s)\right|^{2}+\left.J_{6}(s)\right|^{2}\right) d s \\
& +2 \int_{0}^{t \wedge v_{R}}\left(P_{s}-Q_{s},\left(g\left(r(s), P_{s}, P_{\delta(s)}\right)-g\left(\bar{r}(s), \hat{Z}_{s}, \widetilde{Z}_{s}\right)\right) d W_{s}\right),
\end{aligned}
$$


where

$$
\begin{array}{lrl}
\mu_{3}=A \bar{\beta}^{2}+5 \bar{\alpha}+24 L^{2}+2, & \mu_{4}=2 \bar{\alpha}+8 L^{2}, \\
\mu_{5}=2 \bar{\alpha}+16 L^{2}, \quad \mu_{6}=24 L^{2}, & \mu_{7}=24 L^{2} .
\end{array}
$$

By the Bukholder-Davis-Gundy inequality, the Young inequality and 4.32, we have

$$
\begin{aligned}
\mathbb{E} & {\left[\sup _{0 \leq s \leq t} \int_{0}^{s \wedge \nu_{R}}\left(P_{s}-Q_{s},\left(g\left(r(s), P_{s}, P_{\delta(s)}\right)-g\left(\bar{r}(s), \hat{Z}_{s}, \widetilde{Z}_{s}\right)\right) d W_{s}\right)\right] } \\
\leq & \frac{1}{4} \mathbb{E}\left[\sup _{0 \leq s \leq t}\left|P_{s \wedge \nu_{R}}-Q_{s \wedge \nu_{R}}\right|^{2}\right]+\frac{\mu_{2}}{4} \mathbb{E}\left[\int_{0}^{t \wedge v_{R}}\left\|g\left(r(s), P_{s}, P_{\delta(s)}\right)-g\left(\bar{r}(s), \hat{Z}_{s}, \widetilde{Z}_{s}\right)\right\|_{2}^{2} d s\right] \\
\leq & \frac{1}{4} \mathbb{E}\left[\sup _{0 \leq s \leq t}\left|P_{s \wedge v_{R}}-Q_{s \wedge \nu_{R}}\right|^{2}\right]+\int_{0}^{t \wedge \nu_{R}}\left(2 \mu_{2} L^{2} \mathbb{E}\left\|P_{s}-Q_{s}\right\|_{C}^{2}+2 \mu_{2} L^{2} \mathbb{E}\left\|Q_{s}-\hat{Z}_{s}\right\|_{C}^{2}\right. \\
& \left.+2 \mu_{2} L^{2} \mathbb{E}\left\|P_{\delta(s)}-Q_{\delta(s)}\right\|_{C}^{2}+2 \mu_{2} L^{2} \mathbb{E}\left\|Q_{\delta(s)}-\widetilde{Z}_{s}\right\|_{C}^{2}+\frac{\mu_{2}}{2} \mathbb{E}\left|J_{6}(s)\right|^{2}\right) d s,
\end{aligned}
$$

where $\mu_{2}$ is a positive constant. Combining (4.33) with 4.34], we have

$$
\begin{aligned}
& \mathbb{E}\left[\sup _{0 \leq t \leq t_{1}}\left|P_{t \wedge v_{R}}-Q_{t \wedge v_{R}}\right|^{2}\right] \\
& \leq \mu_{3} \int_{0}^{t_{1} \wedge v_{R}} \mathbb{E}\left[\sup _{0 \leq u \leq s}\left|P_{u}-Q_{u}\right|^{2}\right] d s+\mu_{4} \int_{0}^{t_{1} \wedge v_{R}} \mathbb{E}\left\|Q_{s}-\bar{Z}_{s}\right\|_{C}^{2} d s \\
& +\mu_{5} \int_{0}^{t_{1} \wedge v_{R}} \mathbb{E}\left\|Q_{s}-\hat{Z}_{s}\right\|_{C}^{2} d s+\mu_{6} \int_{0}^{t_{1} \wedge v_{R}} \mathbb{E}\left\|P_{\delta(s)}-Q_{\delta(s)}\right\|_{C}^{2} d s \\
& +\mu_{7} \int_{0}^{t_{1} \wedge v_{R}} \mathbb{E}\left\|Q_{\delta(s)}-\bar{Z}_{\delta(s)}\right\|_{C}^{2} d s+2 \int_{0}^{t \wedge v_{R}}\left(\mathbb{E}\left|J_{4}(s)\right|^{2}+\mathbb{E}\left|J_{5}(s)\right|^{2}+\mathbb{E}\left|J_{6}(s)\right|^{2}\right) d s \\
& +\frac{1}{2} \mathbb{E}\left[\sup _{0 \leq t \leq t_{1}}\left|P_{t \wedge v_{R}}-Q_{t \wedge v_{R}}\right|^{2}\right]+\int_{0}^{t_{1} \wedge v_{R}}\left(4 \mu_{2} L^{2} \mathbb{E}\left\|P_{s}-Q_{s}\right\|_{C}^{2}+4 \mu_{2} L^{2} \mathbb{E}\left\|Q_{s}-\hat{Z}_{s}\right\|_{C}^{2}\right. \\
& \left.+4 \mu_{2} L^{2} \mathbb{E}\left\|P_{\delta(s)}-Q_{\delta(s)}\right\|_{C}^{2}+4 \mu_{2} L^{2} \mathbb{E}\left\|Q_{\delta(s)}-\widetilde{Z}_{s}\right\|_{C}^{2}+\mu_{2} \mathbb{E}\left|J_{6}(s)\right|^{2}\right) d s .
\end{aligned}
$$

By Lemma 4.6, 4.8 and 4.9 , we have

$$
\begin{aligned}
\frac{1}{2} \mathbb{E}\left[\sup _{0 \leq t \leq t_{1}}\left|P_{t \wedge v_{R}}-Q_{t \wedge v_{R}}\right|^{2}\right] \\
\leq\left(\mu_{3}+\mu_{6}+8 \mu_{2} L^{2}\right) \int_{0}^{t_{1}} \mathbb{E}\left[\sup _{0 \leq u \leq s}\left|P_{u \wedge v_{R}}-Q_{u \wedge v_{R}}\right|^{2}\right] d s+\mu_{4} \int_{0}^{t_{1} \wedge v_{R}} \mathbb{E}\left\|Q_{s}-\bar{Z}_{s}\right\|_{C}^{2} d s \\
\quad+\left(\mu_{5}+4 \mu_{2} L^{2}\right) \int_{0}^{t_{1} \wedge v_{R}} \mathbb{E}\left\|Q_{s}-\hat{Z}_{s}\right\|_{C}^{2} d s+\left(\mu_{7}+4 \mu_{2} L^{2}\right) \int_{0}^{t_{1} \wedge v_{R}} \mathbb{E}\left\|Q_{\delta(s)}-\bar{Z}_{\delta(s)}\right\|_{C}^{2} d s \\
\quad+2\left(C_{6}+C_{7}+C_{8}\right) \Delta+\mu_{2} C_{8} \Delta \\
\leq\left(\mu_{3}+\mu_{6}+8 \mu_{2} L^{2}\right) \int_{0}^{t_{1}} \mathbb{E}\left[\sup _{0 \leq u \leq s}\left|P_{u \wedge v_{R}}-Q_{u \wedge v_{R}}\right|^{2}\right] d s \\
+\left(\mu_{4} C_{10} T+\left(\mu_{5}+4 \mu_{2} L^{2}\right) C_{11} T+\left(\mu_{7}+4 \mu_{2} L^{2}\right) C_{12} T+2\left(C_{6}+C_{7}+C_{8}\right)+\mu_{2} C_{8}\right) \Delta .
\end{aligned}
$$

Applying the Gronwall inequality, we obtain

$$
\mathbb{E}\left[\sup _{0 \leq t \leq T}\left|P_{t \wedge v_{R}}-Q_{t \wedge v_{R}}\right|^{2}\right] \leq C_{13} \Delta .
$$


Thus, the proof is complete.

Letting $R \rightarrow \infty$, we have the following theorem.

Theorem 4.11. Suppose that the preceding assumptions hold, then

$$
\lim _{\Delta \rightarrow 0} \mathbb{E}\left[\sup _{0 \leq t \leq T}\left|P_{t}-Q_{t}\right|^{2}\right]=0
$$

Remark 4.12. The result is known without time delay in [19], but it is new in the case of variable delay. As we stated in the Introduction section, so far most of the existing strong convergence result for numerical methods requires the coefficients of the SAPEs to be globally Lipschitz continuous (see, e.g, [19, 22, [23]) and little is yet known about the convergence for numerical solution to SAPEs under the local Lipschitz condition. As sequels to this work, we shall discuss the strong convergence result for numerical methods under the local Lipschitz condition in future work.

\section{Numerical examples}

In this section, we consider the numerical solution and the strong convergence of some SDAPEs with Markovian switching by the $\operatorname{SS} \theta$ method given in (3.4). We use sample average to approximate the expectation. More precisely, we measure the maximum norms errors by

$$
\epsilon=\frac{1}{\hat{M}} \sum_{i=1}^{\hat{M}} \max _{0 \leq n \leq M-1}\left|x^{i}\left(t_{n}\right)-x_{n}^{i}\right|^{2},
$$

where $\hat{M}, x^{i}\left(t_{n}\right), x_{n}^{i}$ denote the number of sample paths, the $i$ th true solution at time $t_{n}$ and the $i$ th numerical solution at time $t_{n}$, respectively. In the following simulations, we set $\hat{M}=1000$.

Table 1: The maximum norms errors of SS $\theta$ and EM method for solving (5.1)

\begin{tabular}{ccccccc}
\hline$\Delta t$ & $\theta=0$ & $\theta=0.2$ & $\theta=0.5$ & $\theta=0.8$ & $\theta=1$ & EM \\
\hline $2^{-4}$ & $*$ & $5.8779 \mathrm{e}-002$ & $5.3161 \mathrm{e}-002$ & $5.2947 \mathrm{e}-002$ & $5.0462 \mathrm{e}-002$ & $*$ \\
$2^{-5}$ & $4.7722 \mathrm{e}-002$ & $4.9001 \mathrm{e}-002$ & $4.7601 \mathrm{e}-002$ & $4.8312 \mathrm{e}-002$ & $4.7538 \mathrm{e}-002$ & $5.0948 \mathrm{e}-002$ \\
$2^{-6}$ & $4.7309 \mathrm{e}-002$ & $4.7939 \mathrm{e}-002$ & $4.7372 \mathrm{e}-002$ & $4.7252 \mathrm{e}-002$ & $4.7173 \mathrm{e}-002$ & $4.9077 \mathrm{e}-002$ \\
$2^{-7}$ & $4.7076 \mathrm{e}-002$ & $4.7545 \mathrm{e}-002$ & $4.6437 \mathrm{e}-002$ & $4.7967 \mathrm{e}-002$ & $4.5905 \mathrm{e}-002$ & $4.6863 \mathrm{e}-002$ \\
\hline
\end{tabular}

Example 5.1. Consider the following SAPEs without Markovian switching as in [11]

$$
\begin{cases}d_{t} P_{t}=\left[-\frac{\partial P_{t}}{\partial a}-\frac{P_{t}}{(1-a)^{2}}-t P_{t}\right] d t+P_{t} d W_{t}, & (t, a) \in(0,1) \times(0,1) \\ P(0, a)=\varphi(a), & a \in(0,1) \\ P(t, 0)=\int_{0}^{1} \frac{P(t, a)}{(1-a)^{2}} d a, & t \in(t, a) \in(0,1) \times(0,1)\end{cases}
$$

where $W_{t}$ is a scalar Brownian motion, with $A=1, T=1, \mu(t, a)=\beta(t, a)=\frac{1}{(1-a)^{2}}, f(t, P)=-t P$, $g(t, P)=P, \varphi=\exp \left(\frac{-1}{1-a}\right)$. Clearly, the operators $f$ and $g$ satisfy conditions $(\mathbb{H} 1)-(\mathbb{H} 2), \mu(t, a)$, 


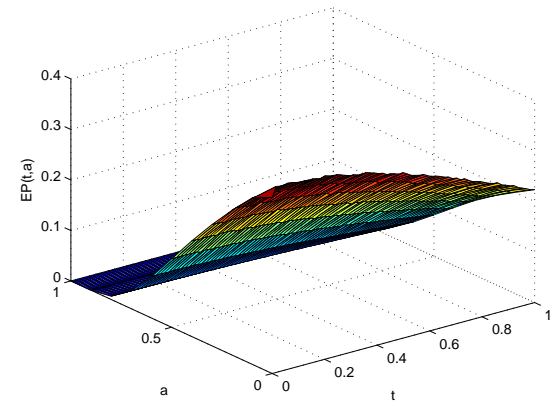

(a) Exact solution without perturbation

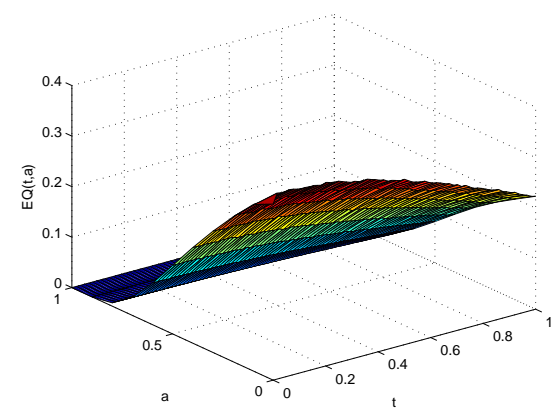

(c) $\operatorname{SS} \theta$ method with $\theta=0.5$

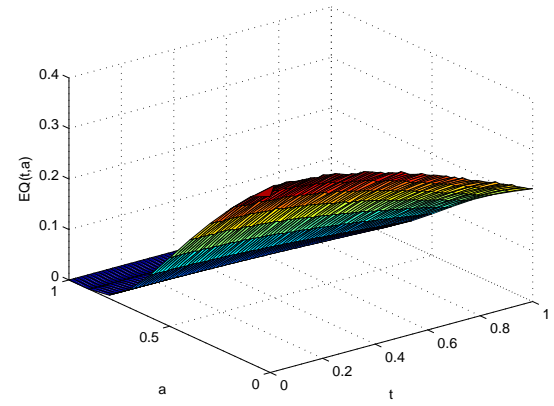

(b) EM method

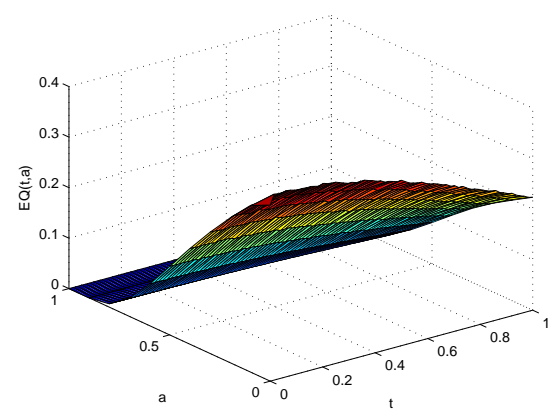

(d) $\mathrm{SS} \theta$ method with $\theta=1$

Fig. 1. Expectation simulations for (5.1)

$\beta(t, a)$ satisfy condition ( $\mathbb{H} 3)$. By Theorem 4.11, the numerical solution will converge to the exact solution in the sense of mean square. Note that the explicit solution to 5.1 without perturbation is

$$
\mathbb{E} P(t, a)=\exp \left(\frac{-1}{1-a}-\frac{t^{2}}{2}\right)
$$

It is difficult to obtain the true explicit solution to 5.1, so the explicit solution to 5.1 can be replaced by

$$
\exp \left(\frac{-1}{1-a}-\frac{t^{2}}{2}\right)\left(1+\Delta W_{t}\right)
$$

(see e.g.,[11, 22, 24]). Setting step size $\Delta t=0.005$ and $\Delta a=0.05$, we simulate the expected value of the exact solution to (5.1) and the numerical solution by EM and SS $\theta$ method with $\theta=0.5, \theta=1$, respectively (see Fig. 1 ), where

$$
\mathbb{E} Q(t, a) \approx \frac{1}{\hat{M}} \sum_{k=1}^{\hat{M}} Q_{k}(t, a) .
$$




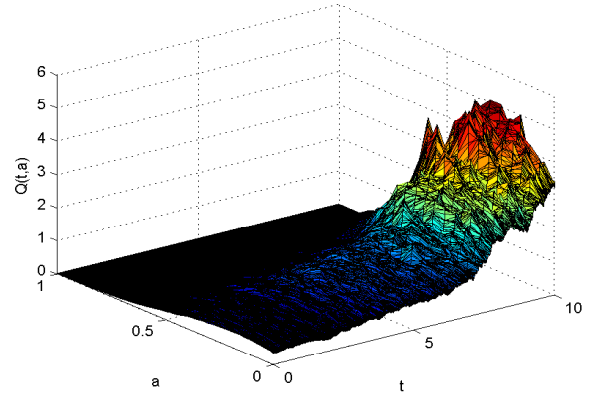

(a) State trajectories of subsystem 1

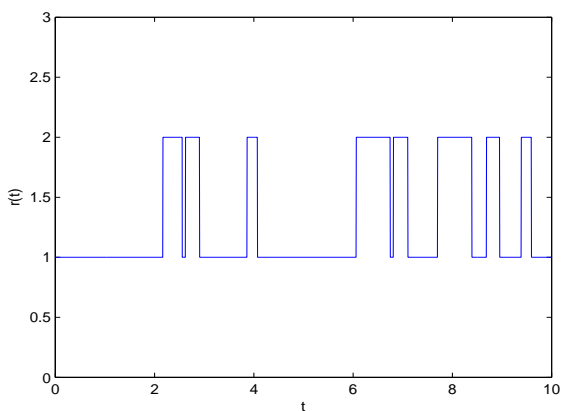

(c) State trajectories of Markov chian $r(t)$

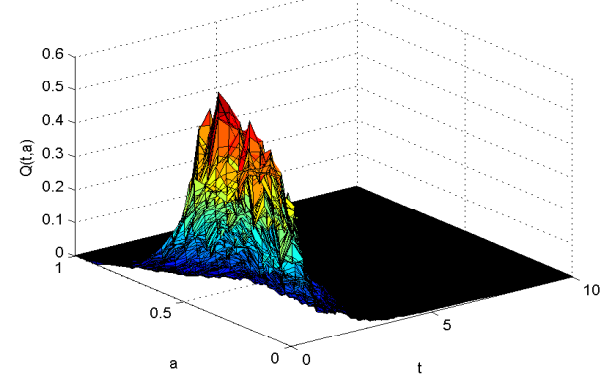

(b) State trajectories of subsystem 2

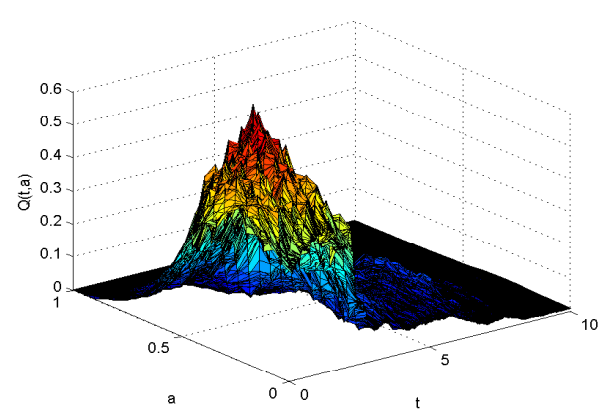

(d) State trajectories of switched system

Fig. 2. Single simulation for 5.2 by $\operatorname{SS} \theta$ method with $\theta=1$

The maximum norms errors of $\mathrm{SS} \theta$ and EM method for solving (5.1) are shown in Table 1 with the same age step size $\Delta a=0.1$. Numerical experiments show the smaller error of $\operatorname{SS} \theta$ method 110 in comparison with the explicit EM method.

Example 5.2. Let $W_{t}$ be a scalar Brownian motion and $r(t)$ be a right-continuous Markov chain taking values in $\mathbb{S}=\{1,2\}$ with generator $\Gamma=\left(\begin{array}{ll}-1 & 1 \\ -2 & 2\end{array}\right)$. SDAPEs with Markovian switching 5.2 are considered to be a class of hybrid systems, which consist of two distinct subsystems, denoted by subsystem 1 and subsystem 2 . Note that subsystem 1 differs from subsystem 2 only in the drift and diffusion coefficients, namely, $f$ and $g$.

$$
\left\{\begin{aligned}
d_{t} P_{t}=[ & \left.-\frac{\partial P_{t}}{\partial a}-\mu(t, a) P_{t}+f\left(r(t), P_{t}, P_{\delta(t)}\right)\right] d t & & \\
& +g\left(r(t), P_{t}, P_{\delta(t)}\right) d W_{t}, & & (t, a) \in(0,3) \times(0,1) \\
P(t, a)= & \varphi(t, a), r(0)=1, & & (t, a) \in[-\tau, 0] \times(0,1) \\
P(t, 0)= & \int_{0}^{1} \beta(t, a) P(t, a) d a, & & (t, a) \in(0,3) \times(0,1)
\end{aligned}\right.
$$




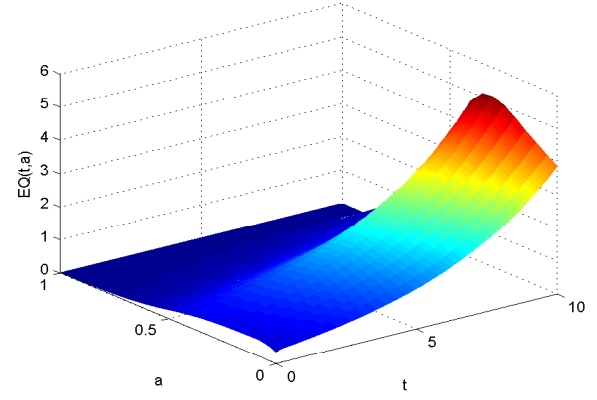

(a) Sample mean of solution to subsystem 1

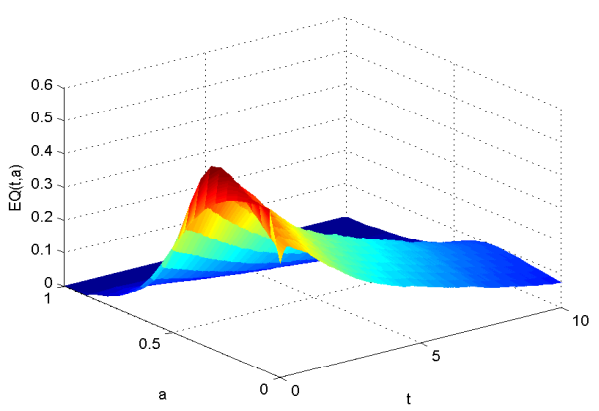

(c) Sample mean of solution to switched system

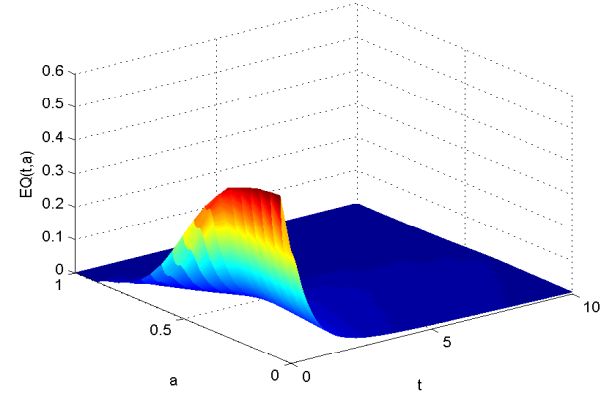

(b) Sample mean of solution to subsystem 2

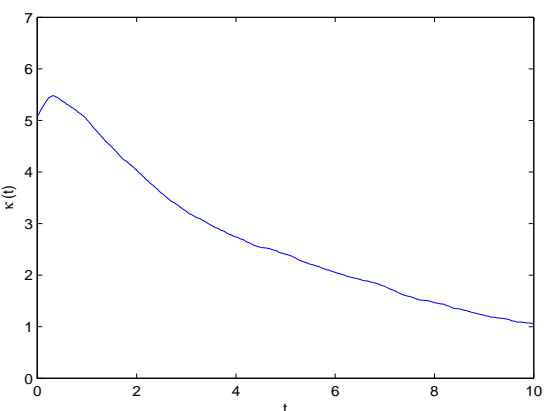

(d) Sample mean of total population for switched system

Fig. 3. Expectation simulations for 5.2 by $\operatorname{SS} \theta$ method with $\theta=1$

where

$$
\begin{array}{cc}
\delta(t)=t-\tau, & \tau=0.1, \\
f(1, x, y)=1.5 x-0.2 y, & g(1, x, y)=0.5 x, \\
f(2, x, y)=-0.5 x+0.1 y, & g(2, x, y)=-\sin x, \\
\beta(t, a)= \begin{cases}2, & \text { if } 0.2 \leq a \leq 0.6 \\
0, & \text { otherwise }\end{cases} \\
\mu(t, a)=\frac{10 \exp (10(a-0.5))}{\exp (10(a-0.5))+\exp (-10(a-0.5))}, \\
\varphi(t, a)=\frac{0.5}{\exp (10(a-0.5))+1} .
\end{array}
$$

For switched system (5.2), we take the birth term $\beta(t, a)$ to have the form 5.6 which means that individuals are fecund if they are not too old or too young. The death modulus (5.7) we used corresponds to a situation where mortality is low until around a certain age, at which point 
mortality increases dramatically (see, e.g., [1, 3]). The initial population distribution (5.8) is an expansive shape, which is typical for fast-growing countries where each birth cohort (a group of people born in the same year or years period ) is larger than the previous one (Latin American, Africa) (see, e.g.,[20, 26]). Obviously, the conditions ( $\mathbb{H} 1)-(\mathbb{H} 3)$ are satisfied. Applying Theorem 4.11, we can deduce that the numerical solution will tend to the exact solution in the mean square sense.

We set $\Delta a=0.05, \Delta t=0.005$ and $\theta=1$. Fig 2.(c) shows the sample trajectories of Markov chian $r(t)$, which determine the switching rule in the single simulation. Fig 2.(a), (b) and (d) give the state trajectories of the solutions to subsystem 1 , subsystem 2 and switched system by $\operatorname{SS} \theta$ method with $\theta=1$, respectively. It seems that subsystem 1 is unstable and subsystem 2 is stable, but the switched system 5.2 is stable. We also plot the sample mean of the solutions to the three systems in Fig 3.(a), (b) and (c), respectively, where $\mathbb{E} Q(t, a)$ is defined in Example 5.1. The sample mean of total population density for switched system 5.2 is illustrated in Fig 3.(d), where total population density $\kappa(t)$ at time $t$ is defined by

$$
\kappa(t)=\sum_{a=0}^{A} \mathbb{E} Q(t, a) .
$$

This clearly reveals the population dynamics tendency.

\section{Acknowledgment}

This work was supported in part by the Natural Science Foundation of China (No. 71571001, 61703003), the Promoting Plan of Higher Education of Anhui Province (TSKJ2015B24) and the Natural Science Foundation of Universities in Anhui Province (KJ2018A0119) .

\section{References}

[1] P.A. Bruce, A variable time step method for an age-dependent population with nonlinear diffusion, SIAM J. Numer. Anal 37 (2000) 1571-1589.

[2] W. Cao, P. Hao, Z. Zhang, Split-step $\theta$-method for stochastic delay differential equations, Applied Numerical Mathematics 76 (2014) 19-33.

[3] S.E. Esipov, J.A. Shapiro, Kinetic model of proteus mirabilis swarm colony development, J. Math. Biol. 36 (1998) 249-268.

[4] Q. Guo, W. Liu, X. Mao, R. Yue, The partially truncated Euler-Maruyama method and its stability and boundedness, Applied Numerical Mathematics 115 (2017) 235-251.

[5] D.J. Higham, Stochastic ordinary differential equations in applied and computational mathematics, IMA Journal of Applied Mathematics 76 (2011) 449-474.

[6] D.J. Higham, P.E. Kloeden, Convergence and stability of implicit methods for jump-diffusion, International Journal of Numerical Analysis and Modeling 3 (2006) 125-140.

[7] D.J. Higham, X. Mao, A.M. Stuart, Strong convergence of Euler-type methods for nonlinear stochastic differential equations, SIAM J. Numer. Anal. 40 (2003) 1041-1063.

140 [8] C. Huang, Exponential mean square stability of numerical methods for systems of stochastic differential equations, Journal of Computational and Applied Mathematics 236 (2012) 4016-4026.

[9] U. Küchler, E. Platen, Strong discrete time approximation of stochastic differential equations with time delay, Math. Comput. Simulation 54 (2000) 189-205.

[10] R. Li, P. Leung, W. Pang, Convergence of numerical solutions to stochastic age-dependent population equations with markovian switching, Journal of Computational and Applied Mathematics 233 (2009) 1046-1055.

[11] R. Li, H. Meng, Q. Chang, Convergence of numerical solutions to stochastic age-dependent population equations, Journal of Computational and Applied Mathematics 193 (2006) 109-120.

[12] F.M.G. Magpantay, N. Kosovalić, An age-structured population model with state-dependent delay: Dynamics, IFAC 48 (2015) 099-104. 
[13] F.M.G. Magpantay, N. Kosovalić, J. Wu, An age-structured population model with state-dependent delay: Derivation and numerical integration, SIAM J. Numer. Anal. 52 (2014) 735-756.

[14] X. Mao, Stochastic Differential Equations and Applications, Harwood, NewYork, 2007.

[15] X. Mao, The truncated Euler-Maruyama method for stochastic differential equations, Journal of Computational and Applied Mathematics 290 (2015) 370-384

16] X. Mao, S. Sabanis, Numerical solutions of stochastic dierential delay equations under local lipschitz condition, Journal of Computational and Applied Mathematics 151 (2003) 215-227.

[17] H. Mo, F. Deng, C. Zhang, Exponential stability of the split-step $\theta$-method for neutral stochastic delay differential equations with jumps, Applied Mathematics and Computation 315 (2017) 85-95.

[18] Y. Pei, H. Yang, Q. Zhang, F. Shen, Asymptotic mean-square boundedness of the numerical solutions of stochastic age-dependent population equations with poisson jumps, Applied Mathematics and Computation 320 (2018) 524534.

[19] A. Rathinasamy, Split-step $\theta$-methods for stochastic age-dependent population equations with markovian switching, Nonlinear Analysis: Real World Applications 13 (2012) 1334-1345.

[20] K. Simona, K. Natasa, B. Vladimir, Clustering of population pyramids, Informatica 32 (2008) 157-167.

165 [21] A.M. Stuart, A.R. Humphries, Dynamical Systems and Numerical Analysis, Cambridge University Press, UK, 1996.

[22] J. Tan, A. Rathinasamy, Y. Pei, Convergence of the split-step $\theta$-method for stochastic age-dependent population equations with poisson jumps, Applied Mathematics and Computation 254 (2015) 305-317.

[23] J. Tan, H. Wang, Convergence and stability of the split-step backward euler method for linear stochastic delay integro-differential equations, Mathematical and Computer Modelling 51 (2010) 504-515.

[24] L. Wang, X. Wang, Convergence of the semi-implicit euler method for stochastic age-dependent population equations with poisson jumps, Applied Mathematical Modelling 34 (2010) 2034-2043.

[25] Z. Yan, A. Xiao, X. Tang, Strong convergence of the split-step theta method for neutral stochastic delay differentia equations, Applied Numerical Mathematics 120 (2017) 215-232.

175 [26] C. Yen, J. Lin, T. Chiu, Comparison of population pyramid and demographic characteristics between people with an intellectual disability and the general population, Research in Developmental Disabilities 34 (2013) 910-915.

[27] C. Yuan, X. Mao, Convergence of the euler maruyama method for stochastic differential equations with markovian switching, Mathematics and Computers in Simulation 64 (2004) 223-235.

[28] Q. Zhang, C. Han, Numerical analysis for stochastic age-dependent population equations, Applied Mathematics and Computation 169 (2005) 278-294.

[29] Q. Zhang, W. Liu, Z. Niu, Existence, uniqueness and exponential stability for stochastic age-dependent population, Applied Mathematics and Computation 154 (2004) 183-201. 